\title{
Exactly solvable Hermite, Laguerre, and Jacobi type quantum parametric oscillators
}

\author{
Şirin A. Büyükaşık and Zehra Çayiç \\ Department of Mathematics, Izmir Institute of Technology, 35430 Urla, Izmir, Turkey
}

(Received 26 May 2016; accepted 3 December 2016; published online 20 December 2016)

\begin{abstract}
We introduce exactly solvable quantum parametric oscillators, which are generalizations of the quantum problems related with the classical orthogonal polynomials of Hermite, Laguerre, and Jacobi type, introduced in the work of Büyükaş1k et al. [J. Math. Phys. 50, 072102 (2009)]. Quantization of these models with specific damping, frequency, and external forces is obtained using the Wei-Norman Lie algebraic approach. This determines the evolution operator exactly in terms of two linearly independent homogeneous solutions and a particular solution of the corresponding classical equation of motion. Then, time-evolution of wave functions and coherent states are found explicitly. Probability densities, expectation values, and uncertainty relations are evaluated and their properties are investigated under the influence of the external terms. Published by AIP Publishing. [http://dx.doi.org/10.1063/1.4972293]
\end{abstract}

\section{INTRODUCTION}

Evolution of quantum systems with time-variable parameters is rather old but still an extensive area of research. One of the most fundamental models with explicitly time-dependent Hamiltonian is probably the quantum harmonic oscillator. It appears in many physical branches such as quantum optics, ${ }^{1-3}$ quantum fluid dynamics, ${ }^{4}$ ion-traps,${ }^{5}$ and cosmology. ${ }^{6}$ It is a useful model also in quantum information ${ }^{7}$ and quantum computation. ${ }^{8}$

As known, for imitation of damped oscillations, Caldirola ${ }^{9}$ and Kanai ${ }^{10}$ introduced a harmonic oscillator with an exponentially increasing mass. This model admits exact solutions and is widely used to study dissipation in quantum mechanics. ${ }^{11}$ Then, different methods have been developed for solving the time-dependent quantum harmonic oscillator. It has been considered first by Husimi, ${ }^{12}$ who by proper ansatz reduced the problem to a non-linear Riccati equation. Then, other methods like the path integral method, ${ }^{13}$ the Lewis-Riesenfeld time invariant method, ${ }^{14}$ and the Wei-Norman dynamical symmetry method ${ }^{15}$ were found. In all these approaches, the solution of the quantum problem is given in terms of the classical one. ${ }^{16-22}$

The generalized time-dependent harmonic oscillator, which contains the mixed term $(\hat{q} \hat{p}+\hat{p} \hat{q})$ and the linear terms $\hat{q}$ and $\hat{p}$ as well, also allows an exact treatment using the several approaches listed above. Husimi, in fact, considered the forced harmonic oscillator and obtained Gaussian type solutions using trial functions. ${ }^{12}$ Integrals of motion and coherent states of a damped and forced parametric oscillator were constructed in Ref. 23. In Ref. 24 the forced Caldirola-Kanai oscillator was solved by the Lewis-Riesenfeld invariant approach, and also by path integration of the Lagrangian giving an exact Feynman propagator. The dynamical invariant approach was used also in Refs. 25-29 to find solutions, uncertainty relations, and coherent states of the generalized oscillator. Recently, quantum integrals of motion for several particular models of the damped and generalized oscillators were constructed in Ref. 30. In Ref. 31 a unitary operator which connects the generalized oscillator to its new reduced form was obtained, and in Ref. 32, the propagators of the general quadratic Hamiltonian systems are obtained from the classical action.

Although formal solutions of the general oscillator are obtained by different approaches, exact solutions are investigated mostly for the driven Caldirola-Kanai oscillator. Recently, a wide class of exactly solvable harmonic oscillator models with specific damping and frequency dependence were introduced in terms of the Sturm-Liouville problems for the classical orthogonal polynomials and hypergeometric functions. ${ }^{33}$ The goal of this work is to extend our previous results and provide 
exact explicit solutions for a large class of general quadratic oscillator models, related with the classical orthogonal polynomials. For this we use the Wei-Norman algebraic approach, ${ }^{15}$ also known as evolution operator approach, which has been used in many works, such as Refs. 34-41. It is a powerful technique for solving evolution problems whose Hamiltonian is a linear combination of generators of a finite dimensional Lie algebra, so that the evolution operator can be represented as product of exponential operators. In case when the Hamiltonian consists of generators of $\operatorname{su}(1,1)$ and Heisenberg-Weyl Lie algebras $(s u(1,1) \bigoplus W)$, finding the evolution operator reduces to solving a nonlinear system of six first-order differential equations, which can be solved by quadrature. In Sec. II, we obtain and express the solution of this system in terms of two linearly independent homogeneous solutions and a particular solution to the corresponding classical equation of motion. Knowing the exact evolution operator we evaluate the corresponding wave functions and probability densities. Then, the evolution of the initially Glauber coherent states, expectation values, and uncertainty relations at these states are computed. Additionally, we show that the results obtained by the evolution operator approach agree with those obtained by the Lewis-Riesenfeld invariant approach in Ref. 27. In Sec. III, we introduce exactly solvable generalized harmonic oscillators related with the classical Hermite, Laguerre, and Jacobi type orthogonal polynomials. These quantum parametric oscillators with specific damping and frequency are investigated under the influence of the external terms, and can be seen as generalization of our previous results. ${ }^{33}$ The original frequency $\omega^{2}(t)$ of these models depends on the eigenvalues of the related Sturm-Liouville problem. As known, the external mixed term parameter modifies the original frequency of the classical oscillator. It happens that special choice of this parameter gives a new frequency $\Omega^{2}(t)$ in terms of a different eigenvalue of the same Sturm-Liouville problem. Then, including also the linear external terms, we call these models as generalized Hermite, Laguerre, and Jacobi type quantum oscillators. For each oscillator type, we give examples with explicit solutions and discuss their properties according to the influence of the external terms. Illustrative plots are constructed for the probability densities and uncertainty relations. Section IV includes brief summary and discussion of the present results.

\section{EVOLUTION OPERATOR AND SOLUTION OF THE GENERALIZED TIME-DEPENDENT HARMONIC OSCILLATOR}

\section{A. Quantization of the generalized quadratic oscillator}

The generalized Hamiltonian for classical oscillator with time-dependent parameters is of the form

$$
H_{g}(x, p, t)=\frac{p^{2}}{2 \mu(t)}+\frac{\mu(t) \omega^{2}(t)}{2} x^{2}+B(t) x p+D(t) x+E(t) p+F(t)
$$

and the corresponding equations of motion are

$$
\begin{gathered}
\dot{x}=\frac{\partial H}{\partial p}=B(t) x+\frac{p}{\mu(t)}+E(t), \\
\dot{p}=-\frac{\partial H}{\partial x}=-\left(\mu(t) \omega^{2}(t) x+B(t) p+D(t)\right) .
\end{gathered}
$$

Then, we have the classical equation of motion in position space

$$
\ddot{x}+\frac{\dot{\mu}}{\mu} \dot{x}+\left(\omega^{2}(t)-\left(\dot{B}+B^{2}+\frac{\dot{\mu}}{\mu} B\right)\right) x=-\frac{1}{\mu} D+\dot{E}+\left(\frac{\dot{\mu}}{\mu}+B\right) E
$$

and the oscillator equation in momentum space

$$
\ddot{p}-\frac{\left(\mu \dot{\omega}^{2}\right)}{\mu \omega^{2}} \dot{p}+\left(\omega^{2}(t)+\left(\dot{B}-B^{2}-\frac{\left(\mu \dot{\omega}^{2}\right)}{\mu \omega^{2}} B\right)\right) p=-\dot{D}+\left(\frac{\left(\mu \dot{\omega}^{2}\right)}{\mu \omega^{2}}+B\right) D-\mu \omega^{2} E .
$$

We notice that the parameter $B(t)$ of the mixed term in Hamiltonian (1) leads to modification of the original frequency $\omega^{2}(t)$, and the external parameters $B(t), D(t)$, and $E(t)$ all contribute to the 
forcing term of the oscillator. Replacing the canonical variables in classical Hamiltonian (1) by the quantum operators,

$$
x \rightarrow \hat{q}, \quad p \rightarrow \hat{p}, \quad x p \rightarrow \frac{\hat{p} \hat{q}+\hat{q} \hat{p}}{2},
$$

we consider the evolution problem for the quantum harmonic oscillator

$$
\begin{gathered}
i \hbar \frac{\partial}{\partial t} \Psi(q, t)=\hat{H}_{g}(t) \Psi(q, t), \\
\Psi\left(q, t_{0}\right)=\Psi_{0}(q), \quad-\infty<q<\infty,
\end{gathered}
$$

with general quadratic Hamiltonian

$$
\hat{H}_{g}(t)=\frac{\hat{p}^{2}}{2 \mu(t)}+\frac{\mu(t) \omega^{2}(t)}{2} \hat{q}^{2}+\frac{B(t)}{2}(\hat{q} \hat{p}+\hat{p} \hat{q})+D(t) \hat{q}+E(t) \hat{p}+F(t) \hat{I},
$$

where $\mu(t)>0, \omega^{2}(t), B(t), D(t), E(t)$, and $F(t)$ are sufficiently smooth, real-valued parameters depending on time. We find the corresponding evolution operator by the Wei-Norman Lie algebraic approach. Indeed, the Hamiltonian (9) can be written as a time-dependent linear combination of Lie algebra generators as

$$
\hat{H}_{g}(t)=-i\left(\frac{\hbar^{2}}{\mu(t)} \hat{K}_{-}+\mu(t) \omega^{2}(t) \hat{K}_{+}+2 \hbar B(t) \hat{K}_{0}+D(t) \hat{E}_{1}+\hbar E(t) \hat{E}_{2}+F(t) \hat{E}_{3}\right),
$$

where

$$
\hat{E}_{1}=i q, \quad \hat{E}_{2}=\frac{\partial}{\partial q}, \quad \hat{E}_{3}=i \hat{I}
$$

are generators of the Heisenberg-Weyl algebra, and

$$
\hat{K}_{-}=-\frac{i}{2} \frac{\partial^{2}}{\partial q^{2}}, \quad \hat{K}_{+}=\frac{i}{2} q^{2}, \quad \hat{K}_{0}=\frac{1}{2}\left(q \frac{\partial}{\partial q}+\frac{1}{2}\right)
$$

are generators of the $s u(1,1)$ algebra. Then, the evolution operator for the general oscillator can be written as product of exponential operators

$$
\hat{U}_{g}\left(t, t_{0}\right)=\hat{U}_{E}\left(t, t_{0}\right) \hat{U}_{K}\left(t, t_{0}\right),
$$

where

$$
\hat{U}_{E}\left(t, t_{0}\right) \equiv e^{c(t) \hat{E}_{3}} e^{\frac{a(t)}{\hbar} \hat{E}_{1}} e^{-b(t) \hat{E}_{2}}, \quad \hat{U}_{K}\left(t, t_{0}\right) \equiv e^{f(t) \hat{K}_{+}} e^{2 h(t) \hat{K}_{0}} e^{g(t) \hat{K}_{-}},
$$

and $f(t), g(t), h(t), a(t), b(t), c(t)$ are unknown real-valued functions to be determined from the initial value problem (IVP) defining the unitary operator $\hat{U}_{g}$, that is,

$$
\begin{aligned}
i \hbar \frac{d}{d t} \hat{U}_{g}\left(t, t_{0}\right) & =\hat{H}_{g}(t) \hat{U}_{g}\left(t, t_{0}\right), \\
\hat{U}_{g}\left(t_{0}, t_{0}\right) & =\hat{I} .
\end{aligned}
$$

After performing time differentiation and comparing both sides of the operator Equation (13), we obtain that $\hat{U}_{g}\left(t, t_{0}\right)$ is the solution of the problem, if the unknown functions satisfy the nonlinear system of six first-order equations 


$$
\begin{aligned}
\dot{f}+\frac{\hbar}{\mu(t)} f^{2}+2 B(t) f+\frac{\mu(t) \omega^{2}(t)}{\hbar} & =0, & & f\left(t_{0}\right)=0, \\
\dot{g}+\frac{\hbar}{\mu(t)} e^{2 h} & =0, & & g\left(t_{0}\right)=0, \\
\dot{h}+\frac{\hbar}{\mu(t)} f+B(t) & =0, & h\left(t_{0}\right) & =0 . \\
\dot{a}+B(t) a+\mu(t) \omega^{2}(t) b+D(t) & =0, & & a\left(t_{0}\right)=0, \\
\dot{b}-B(t) b-\frac{1}{\mu(t)} a-E(t) & =0, & b\left(t_{0}\right) & =0, \\
\dot{c}+\frac{1}{2 \hbar \mu(t)} a^{2}+\frac{E(t)}{\hbar} a-\frac{\mu(t) \omega^{2}(t)}{2 \hbar} b^{2}+\frac{F(t)}{\hbar} & =0, & & c\left(t_{0}\right)=0 .
\end{aligned}
$$

In fact, (14) and (15) are two independent systems, one for $f, g, h$ and second for $a, b, c$. System (14) can be easily solved by realizing that first line is an initial value problem for the non-linear Riccatti equation, and using substitution $f(t)=\mu(t)(\dot{x} / x-B(t)) / \hbar$, it transforms to the classical homogeneous equation of motion

$$
\ddot{x}+\frac{\dot{\mu}}{\mu} \dot{x}+\left(\omega^{2}(t)-\left(\dot{B}+B^{2}+\frac{\dot{\mu}}{\mu} B\right)\right) x=0,
$$

with initial conditions

$$
x\left(t_{0}\right)=x_{0} \neq 0, \quad \dot{x}\left(t_{0}\right)=x_{0} B\left(t_{0}\right),
$$

whose solution we denote by $x_{1}(t)$. Assuming that all coefficients in Eq. (16) are continuous on a time interval containing $t_{0}$, by $x_{2}(t)$ we denote a second solution of Eq. (16), satisfying the initial conditions

$$
x_{2}\left(t_{0}\right)=0, \dot{x}_{2}\left(t_{0}\right)=1 / \mu\left(t_{0}\right) x_{1}\left(t_{0}\right) .
$$

Then, $g(t)$ can be expressed in terms of these two independent solutions. This gives the solution of system (14) in terms of two linearly independent solutions $x_{1}(t)$ and $x_{2}(t)$ of the homogeneous equation as

$$
\begin{aligned}
& f(t)=\frac{\mu(t)}{\hbar}\left(\frac{\dot{x}_{1}(t)}{x_{1}(t)}-B(t)\right), \\
& g(t)=-\hbar x_{1}^{2}\left(t_{0}\right)\left(\frac{x_{2}(t)}{x_{1}(t)}\right), \\
& h(t)=-\ln \left|\frac{x_{1}(t)}{x_{1}\left(t_{0}\right)}\right| .
\end{aligned}
$$

On the other hand, we realize that in system (15), the equations for $b(t)$ and $a(t)$ are same with the classical equations (2) and (3) for $x(t)$ and $p(t)$, respectively. Then, using first two equations in system (15), we obtain that $b(t)$ is the solution of the nonhomogeneous Eq. (4), with initial conditions

$$
x\left(t_{0}\right)=0, \dot{x}\left(t_{0}\right)=E\left(t_{0}\right),
$$

and we denote this solution by $b(t)=x_{p}(t)$. Similarly, it follows that $a(t)=p_{p}(t)$, where $p_{p}(t)$ is solution of the nonhomogeneous Eq. (5) for momentum, with initial conditions

$$
p\left(t_{0}\right)=0, \dot{p}\left(t_{0}\right)=-D\left(t_{0}\right) .
$$

Then, solution of system (15) is found in terms of the two particular solutions, $x_{p}$ and $p_{p}$, as

$$
\begin{aligned}
& a(t)=p_{p}(t), \\
& b(t)=x_{p}(t), \\
& c(t)=\int_{t_{0}}^{t}\left[\frac{-\left(p_{p}(s)\right)^{2}}{2 \hbar \mu(s)}-\frac{E(s)}{\hbar} p_{p}(s)+\frac{\mu(s) \omega^{2}(s)}{2 \hbar} x_{p}^{2}(s)-\frac{F(s)}{\hbar}\right] d s .
\end{aligned}
$$


Writing $p_{p}(t)$ in terms of $x_{p}(t)$, the solution of this system becomes

$$
\begin{aligned}
a(t)= & \mu(t)\left(\dot{x}_{p}(t)-B(t) x_{p}(t)-E(t)\right), \\
b(t)= & x_{p}(t), \\
c(t)= & \frac{-1}{2 \hbar} \int_{t_{0}}^{t} \mu(s)\left[\dot{x}_{p}^{2}(s)-2 B(s) x_{p}(s) \dot{x}_{p}(s)\right. \\
& \left.+\left(B^{2}(s)-\omega^{2}(s)\right) x_{p}^{2}(s)-E^{2}(s)+\frac{2}{\mu(s)} F(s)\right] d s,
\end{aligned}
$$

showing that solution of the general oscillator is completely determined by solutions $x_{1}(t), x_{2}(t)$, and $x_{p}(t)$ of the classical oscillator. We note that choosing different orderings of the exponential operators in the evolution operator (11) leads to different formulations of the system for the six unknown parameters. In any case, the system can be solved by quadrature, but we cannot always easily see its solution in terms of $x_{1}, x_{2}$, and $x_{p}$, as in the present case. Now, after finding all unknown functions in (11), the exact form of the evolution operator becomes

$$
\begin{aligned}
\hat{U}_{g}\left(t, t_{0}\right)= & \exp \left(\frac{i}{\hbar} \int_{t_{0}}^{t}\left[\frac{-1}{2 \mu(s)} p_{p}^{2}(s)-E(s) p_{p}(s)+\frac{\mu(s) \omega^{2}(s)}{2} x_{p}^{2}(s)-F(s)\right] d s\right) \\
& \times \exp \left(i p_{p}(t) q\right) \times \exp \left(-x_{p}(t) \frac{\partial}{\partial q}\right) \times \exp \left(i \frac{\mu(t)}{2 \hbar}\left(\frac{\dot{x}_{1}(t)}{x_{1}(t)}-B(t)\right) q^{2}\right) \\
& \times \exp \left(\ln \left|\frac{x_{1}\left(t_{0}\right)}{x_{1}(t)}\right|\left(q \frac{\partial}{\partial q}+\frac{1}{2}\right)\right) \times \exp \left(\frac{i}{2} \hbar x_{1}^{2}\left(t_{0}\right)\left(\frac{x_{2}(t)}{x_{1}(t)}\right) \frac{\partial^{2}}{\partial q^{2}}\right),
\end{aligned}
$$

where $p_{p}(t)=\mu(t)\left(\dot{x}_{p}(t)-B(t) x_{p}(t)-E(t)\right)$. Therefore, with this evolution operator we can solve the quantum oscillator problem (7) for given initial data. Here, as initial functions we choose the normalized eigenstates of the standard Hamiltonian,

$$
\varphi_{k}(q)=N_{k} e^{-\frac{m \omega_{0}}{2 \hbar} q^{2}} H_{k}\left(\sqrt{\frac{m \omega_{0}}{\hbar}} q\right)
$$

where $H_{k}\left(\sqrt{m \omega_{0} / \hbar} q\right)$ are the Hermite polynomials, $N_{k}=\left(2^{k} k !\right)^{-1 / 2}\left(m \omega_{0} / \pi \hbar\right)^{1 / 4}$ are normalization constants, and eigenvalues are $E_{k}=\left(\hbar / \omega_{0}\right)(k+1 / 2), k=0,1,2, \ldots$. Applying the evolution operator, and using that $(d / d t)\left(x_{2}(t) / x_{1}(t)\right)=1 /\left(\mu(t) x_{1}^{2}(t)\right)$, we obtain the exact wave function in the form

$$
\begin{aligned}
& \Psi_{k}(q, t)=N_{k} \sqrt{R_{B}(t)} \times \exp \left[i\left(k+\frac{1}{2}\right) \eta(t)\right] \\
& \times \exp \left[-\frac{i}{2 \hbar} \mu(t)\left(B(t)+\frac{\dot{R}_{B}(t)}{R_{B}(t)}\right)\left(q-x_{p}(t)\right)^{2}+\frac{i}{\hbar} p_{p}(t) q\right] \\
& \times \exp \left[\frac{-i}{2 \hbar} \int_{t_{0}}^{t} \mu(s)\left[\dot{x}_{p}^{2}(s)-2 B(s) x_{p}(s) \dot{x}_{p}(s)+\left(B^{2}(s)-\omega^{2}(s)\right) x_{p}^{2}(s)-E^{2}(s)+\frac{2 F(s)}{\mu(s)}\right] d s\right] \\
& \times \exp \left[-\frac{1}{2}\left(\sqrt{\frac{m \omega_{0}}{\hbar}} R_{B}(t)\left(q-x_{p}(t)\right)\right)^{2}\right] \times H_{k}\left(\sqrt{\frac{m \omega_{0}}{\hbar}} R_{B}(t)\left(q-x_{p}(t)\right)\right)
\end{aligned}
$$

where

$$
\eta(t)=\arctan \left(-m \omega_{0} x_{1}^{2}\left(t_{0}\right)\left(\frac{x_{2}(t)}{x_{1}(t)}\right)\right)=-m \omega_{0} \int_{t_{0}}^{t} \frac{R_{B}^{2}(s)}{\mu(s)} d s
$$

and

$$
R_{B}(t)=\sqrt{\frac{x_{0}^{2}}{x_{1}^{2}(t)+\left(m \omega_{0} x_{0}^{2} x_{2}(t)\right)^{2}}} .
$$


The probability density is then

$$
\rho_{k}(q, t)=N_{k}^{2} R_{B}(t) \exp \left(-\left(\sqrt{\frac{m \omega_{0}}{\hbar}} R_{B}(t)\left(q-x_{p}(t)\right)\right)^{2}\right) \times H_{k}^{2}\left(\sqrt{\frac{m \omega_{0}}{\hbar}} R_{B}(t)\left(q-x_{p}(t)\right)\right),
$$

where $R_{B}(t)$ is the squeezing (or spreading) coefficient, and $x_{p}(t)$ is the displacement of the wave packet. We note that $R_{B}(t)$ depends on the mixed term coefficient $B(t)$, but does not depend on the external term parameters $D(t), E(t)$, and $F(t)$. On the other hand, the displacement $x_{p}(t)$ clearly depends on all parameters of the Hamiltonian. By previous assumptions, $x_{1}(t)$ and $x_{2}(t)$ are smooth and cannot be simultaneously zero, and if $x_{p}(t)$ is also smooth, probability density is a conserved quantity, that is

$$
\int_{-\infty}^{\infty} \rho_{k}(q, t) d q=1
$$

In the limiting case, when all external terms are zero, i.e., $B=D=E=F=0$, the probability density takes the form

$$
\rho_{k}(q, t)=N_{k}^{2} \times R_{0}(t) \times \exp \left(-\left(\sqrt{\frac{m \omega_{0}}{\hbar}} R_{0}(t) q\right)^{2}\right) \times H_{k}^{2}\left(\sqrt{\frac{m \omega_{0}}{\hbar}} R_{0}(t)(q)\right),
$$

which coincides with our earlier result in Ref. 33. We also note that $\varrho(t)=1 /\left(\sqrt{m \omega_{0}} R_{B}(t)\right)$ is solution of the Ermakov-Pinney differential equation

$$
\ddot{\varrho}+\frac{\dot{\mu}}{\mu} \dot{\varrho}+\left(\omega^{2}(t)-\left(\dot{B}+B^{2}+\frac{\dot{\mu}}{\mu} B\right)\right) \varrho=\frac{1}{\mu^{2} \varrho^{3}},
$$

with the initial conditions

$$
\varrho\left(t_{0}\right)=\frac{1}{\sqrt{m \omega_{0}}}, \quad \dot{\varrho}\left(t_{0}\right)=\frac{B\left(t_{0}\right)}{\sqrt{m \omega_{0}}} .
$$

Then it is easy to see that, for this particular solution $\varrho(t)$, the wave functions (25) agree with the wave functions obtained by the Lewis-Riesenfeld approach in Ref. 27.

\section{B. Heisenberg picture, expectation, and uncertainties at states $\Psi_{k}(q, t)$.}

The position and momentum operators in Heisenberg picture defined by

$$
\begin{array}{ll}
\hat{q}_{H}(t)=\hat{U}_{g}^{\dagger}\left(t, t_{0}\right) \hat{q}_{S} \hat{U}_{g}\left(t, t_{0}\right), & \hat{q}_{H}\left(t_{0}\right)=\hat{q}_{S}, \\
\hat{p}_{H}(t)=\hat{U}_{g}^{\dagger}\left(t, t_{0}\right) \hat{p}_{S} \hat{U}_{g}\left(t, t_{0}\right), & \hat{p}_{H}\left(t_{0}\right)=\hat{p}_{S}
\end{array}
$$

are obtained explicitly using the evolution operator (23) as

$$
\begin{aligned}
& \hat{q}_{H}(t)=\frac{1}{x_{0}} x_{1}(t) \hat{q}_{H}\left(t_{0}\right)+x_{0} x_{2}(t) \hat{p}_{H}\left(t_{0}\right)+x_{p}(t), \\
& \hat{p}_{H}(t)=\frac{1}{x_{0}} \mu(t)\left(\dot{x}_{1}(t)-B(t) x_{1}(t)\right) \hat{q}_{H}\left(t_{0}\right)+x_{0} \mu(t)\left(\dot{x}_{2}(t)-B(t) x_{2}(t)\right) \hat{p}_{H}\left(t_{0}\right)+p_{p}(t) .
\end{aligned}
$$

These operators satisfy the Heisenberg equations of motion,

$$
\begin{gathered}
\frac{d}{d t} \hat{q}_{H}(t)=\frac{\hat{p}_{H}(t)}{\mu(t)}+B(t) \hat{q}_{H}(t)+E(t), \\
\frac{d}{d t} \hat{p}_{H}(t)=-\left(\mu(t) \omega^{2}(t) \hat{q}_{H}(t)+B(t) \hat{p}_{H}(t)+D(t)\right),
\end{gathered}
$$

where $\hat{q}_{H}(t)$ is the solution of the classical equation (4) and $\hat{p}_{H}(t)$ is the solution of (5). Then, the expectations of position and momentum at state $\Psi_{k}(q, t)$ can be found using

$$
\begin{aligned}
&\langle\hat{q}\rangle_{k}(t) \equiv\left\langle\Psi_{k}(q, t)\left|\hat{q}_{S}\right| \Psi_{k}(q, t)\right\rangle=\left\langle\varphi_{k}(q)\left|\hat{q}_{H}(t)\right| \varphi_{k}(q)\right\rangle, \\
&\langle\hat{p}\rangle_{k}(t) \equiv\left\langle\Psi_{k}(q, t)\left|\hat{p}_{S}\right| \Psi_{k}(q, t)\right\rangle=\left\langle\varphi_{k}(q)\left|\hat{p}_{H}(t)\right| \varphi_{k}(q)\right\rangle,
\end{aligned}
$$


where $\hat{q}_{H}(t)$ is given by (32) and $\hat{p}_{H}(t)$ is given by (33). Indeed, since

$$
\hat{q}_{H}\left(t_{0}\right)=\sqrt{\hbar /\left(2 m \omega_{0}\right)}\left(\hat{a}+\hat{a}^{\dagger}\right), \quad \hat{p}_{H}\left(t_{0}\right)=-i \sqrt{\left(m \omega_{0} \hbar\right) / 2}\left(\hat{a}-\hat{a}^{\dagger}\right),
$$

where $\hat{a}, \hat{a}^{\dagger}$ are lowering and raising operators of the standard Hamiltonian $\hat{H}_{0}=\hbar \omega_{0}\left(\hat{a}^{\dagger} \hat{a}+1 / 2\right)$, and $\hat{a} \varphi_{k}(q)=\sqrt{k} \varphi_{k-1}, \hat{a}^{\dagger} \varphi_{k}(q)=\sqrt{k+1} \varphi_{k+1}, k=0,1,2, \ldots$, we obtain

$$
\langle\hat{q}\rangle_{k}(t)=x_{p}(t), \quad\langle\hat{p}\rangle_{k}(t)=p_{p}(t) .
$$

Then, the expectation values of squares of position and momentum are

$$
\begin{gathered}
\left\langle\hat{q}^{2}\right\rangle_{k}(t)=\left(k+\frac{1}{2}\right)\left(\frac{\hbar}{m \omega_{0} R_{B}^{2}(t)}\right)+x_{p}^{2}(t) \\
\left\langle\hat{p}^{2}\right\rangle_{k}(t)=\left(k+\frac{1}{2}\right)\left(m \omega_{0} \hbar R_{B}^{2}(t)\right)\left[1+\frac{\mu^{2}(t)}{\left(m \omega_{0} R_{B}^{2}(t)\right)^{2}}\left(\frac{\dot{R}_{B}(t)}{R_{B}(t)}+B(t)\right)^{2}\right]+p_{p}^{2}(t) .
\end{gathered}
$$

As a result, the fluctuations for $\hat{q}$ and $\hat{p}$ are found as

$$
\begin{aligned}
& (\Delta \hat{q})_{k}(t)=\sqrt{\left(k+\frac{1}{2}\right)\left(\frac{\hbar}{m \omega_{0} R_{B}^{2}(t)}\right)}, \\
& (\Delta \hat{p})_{k}(t)=\sqrt{\left(k+\frac{1}{2}\right)\left(m \omega_{0} \hbar R_{B}^{2}(t)\right)} \sqrt{1+\frac{\mu^{2}(t)}{\left(m \omega_{0} R_{B}^{2}(t)\right)^{2}}\left(\frac{\dot{R}_{B}(t)}{R_{B}(t)}+B(t)\right)^{2}},
\end{aligned}
$$

and the uncertainty relation in state $\Psi_{k}(q, t)$ becomes

$$
(\Delta \hat{q})_{k}(\Delta \hat{p})_{k}(t)=\hbar\left(k+\frac{1}{2}\right) \sqrt{1+\frac{\mu^{2}(t)}{\left(m \omega_{0} R_{B}^{2}(t)\right)^{2}}\left(\frac{\dot{R}_{B}(t)}{R_{B}(t)}+B(t)\right)^{2}}, k=0,1,2, \ldots
$$

\section{Coherent states, expectation values, and uncertainty relation}

Coherent states of standard harmonic oscillator (Glauber coherent states) have many useful physical and mathematical properties, and they can be defined in different, but equivalent ways. ${ }^{42-44,18}$ As minimum uncertainty states they satisfy $(\Delta \hat{q})_{\alpha}(\Delta \hat{p})_{\alpha}=\hbar / 2$, and are therefore closest to the classical states. Displacement operator coherent states are defined by the action of the displacement operator $D(\alpha)=e^{\alpha \hat{a}^{\dagger}-\alpha^{*} \hat{a}}$ on the ground state, confirming that they are shifted Gaussian wave packets. Coherent states are known also as annihilation operator eigenstates, that is, if $\hat{a}$ is the annihilation operator of the standard Hamiltonian $\hat{H}_{0}$, the coherent states satisfy $\hat{a} \phi_{\alpha}(q)=\alpha \phi_{\alpha}(q)$, for any complex number $\alpha=\alpha_{1}+i \alpha_{2}, \alpha_{1}, \alpha_{2}-$ real. Then, they can be expanded in terms of the eigenstates $\varphi_{k}(q)$ of $\hat{H}_{0}$ as

$$
\phi_{\alpha}(q)=e^{-|\alpha|^{2} / 2} \sum_{k=0}^{\infty} \frac{\alpha^{k}}{\sqrt{k !}} \varphi_{k}(q)
$$

or can be written in a closed form

$$
\phi_{\alpha}(q)=\left(\frac{m \omega_{0}}{\pi \hbar}\right)^{1 / 4} e^{-\frac{i}{2 \hbar}\langle\hat{q}\rangle_{\alpha}\langle\hat{p}\rangle_{\alpha}} e^{\frac{i}{\hbar}\langle\hat{p}\rangle_{\alpha} q} e^{-\frac{m \omega_{0}}{2 \hbar}\left(q-\langle\hat{q}\rangle_{\alpha}\right)^{2}},
$$

where $\langle\hat{q}\rangle_{\alpha}=\sqrt{2 \hbar /\left(m \omega_{0}\right)} \alpha_{1},\langle\hat{p}\rangle_{\alpha}=\sqrt{2 m \omega_{0} \hbar} \alpha_{2}$. According to this, time-evolved coherent states for the standard oscillator become

$$
\phi_{\alpha}(q, t)=e^{-\frac{i \omega}{2} t} e^{-|\alpha(t)|^{2} / 2} \sum_{k=0}^{\infty} \frac{\alpha(t)^{k}}{\sqrt{k !}} \varphi_{k}(q),
$$

where $\alpha(t)=e^{-i \omega t} \alpha\left(t_{0}\right)$. Thus, they are also eigenstates of the annihilation operator, but corresponding to the time-dependent eigenvalues, since $\hat{a} \phi_{\alpha}(q, t)=\alpha(t) \phi_{\alpha}(q, t)$. It follows that coherent states of the standard oscillator remain coherent under time-evolution and they are non-spreading 
wave packets since $d(\Delta \hat{q})_{\alpha}(t) / d t=0$ and expectation of position $\langle\hat{q}\rangle_{\alpha}(t)$ follows the classical trajectory.

Now, we consider time-evolution of initially Glauber coherent states under the influence of the evolution operator (23) of the generalized parametric oscillator, that is, $\Phi_{\alpha}(q, t)=\hat{U}_{g}\left(t, t_{0}\right) \phi_{\alpha}\left(q, t_{0}\right)$. Using that $\phi_{\alpha}\left(q, t_{0}\right)=\phi_{\alpha}(q)$ is given by (35), and $\hat{U}_{g}\left(t, t_{0}\right) \varphi_{k}(q)=\Psi_{k}(q, t)$, one directly gets the generalized coherent states in terms of the wave functions (25),

$$
\Phi_{\alpha}(q, t)=e^{-\frac{|\alpha|^{2}}{2}} \sum_{k=0}^{\infty} \frac{\alpha^{k}}{\sqrt{k !}} \Psi_{k}(q, t) .
$$

Or applying the evolution operator $\hat{U}_{g}\left(t, t_{0}\right)$ to $(36)$, time-evolved coherent states are found explicitly

$$
\begin{aligned}
\Phi_{\alpha}(q, t)= & \left(\frac{m \omega_{0}}{\pi \hbar}\right)^{1 / 4} R_{B}(t) \times \sqrt{\frac{x_{1}(t)}{x_{0}}-i\left(m \omega_{0} x_{0}\right) x_{2}(t)} \\
& \times \exp \left[\frac{i}{\hbar} \int_{t_{0}}^{t}\left(\frac{-1}{2 \mu(s)} p_{p}^{2}(s)-E(s) p_{p}(s)+\frac{\mu(s) \omega^{2}(s)}{2} x_{p}^{2}(s)-F(s)\right) d s\right] \\
& \times \exp \left[-i\left(m \omega_{0}\right) x_{2}(t) R_{B}^{2}(t)\left(x_{1}(t)-i\left(m \omega_{0} x_{0}^{2}\right) x_{2}(t)\right) \alpha^{2}+\frac{\alpha^{2}-|\alpha|^{2}}{2}\right] \\
& \times \exp \left(\frac{i}{\hbar} p_{p}(t) q\right) \times \exp \left[\frac{-i}{2 \hbar} \mu(t)\left(B(t)+\frac{\dot{R}_{B}(t)}{R_{B}(t)}\right)\left(q-x_{p}(t)\right)^{2}\right] \\
& \times \exp \left\{-R_{B}^{2}(t)\left[\sqrt{\frac{m \omega_{0}}{2 \hbar}}\left(q-x_{p}(t)\right)-\left(\frac{x_{1}(t)}{x_{0}}-i\left(m \omega_{0} x_{0}\right) x_{2}(t)\right) \alpha\right]^{2}\right\},
\end{aligned}
$$

and the corresponding probability densities become

$$
\begin{aligned}
& \rho_{\alpha}(q, t)=\sqrt{\frac{m \omega_{0}}{\pi \hbar}} R_{B}(t) \\
& \times \exp \left\{-\left(\frac{m \omega_{0}}{\hbar}\right) R_{B}^{2}(t)\left[q-\left(\sqrt{\frac{2 \hbar}{m \omega_{0}}}\left(\alpha_{1} \frac{x_{1}(t)}{x_{0}}+\alpha_{2}\left(m \omega_{0} x_{0}\right) x_{2}(t)\right)+x_{p}(t)\right)\right]^{2}\right\} .
\end{aligned}
$$

As another approach, we show that coherent states $\Phi_{\alpha}(q, t)$ can be defined also as eigenstates of the annihilation operator $\hat{A}_{0}(t)$ of a certain dynamical invariant $\hat{I}_{0}(t)$ of the generalized Hamiltonian system. Indeed, let $\hat{A}_{0}(t)=\hat{U}_{g}\left(t, t_{0}\right) \hat{a} \hat{U}_{g}^{\dagger}\left(t, t_{0}\right)$ and $\hat{A}_{0}^{\dagger}(t)=\hat{U}_{g}\left(t, t_{0}\right) \hat{a}^{\dagger} \hat{U}_{g}^{\dagger}\left(t, t_{0}\right)$, where $\hat{a}$ and $\hat{a}^{\dagger}$ are the annihilation and creation operators for $\hat{H}_{0}$, respectively. Then the operator $\hat{I}_{0}(t)$, defined as

$$
\hat{I}_{0}(t)=\hbar\left(\hat{A}_{0}^{\dagger}(t) \hat{A}_{0}(t)+\frac{1}{2}\right)
$$

satisfies

$$
\frac{d \hat{I}_{0}}{d t} \equiv \frac{\partial \hat{I}_{0}}{\partial t}+\frac{1}{i \hbar}\left[\hat{I}_{0}, \hat{H}_{g}\right]=0,
$$

and is therefore an invariant for the system. Knowing the evolution operator, the lowering $\hat{A}_{0}(t)$ and raising $\hat{A}_{0}^{\dagger}(t)$ operators are explicitly found as

$$
\begin{aligned}
& \hat{A}_{0}(t)=e^{i \eta(t)}\left\{\left[\sqrt{\frac{m \omega_{0}}{2 \hbar}} R_{B}(t)+\frac{i \mu(t)}{\sqrt{2 m \omega_{0} \hbar} R_{B}(t)}\left(B(t)+\frac{\dot{R}_{B}(t)}{R_{B}(t)}\right)\right]\left(\hat{q}-x_{p}(t)\right)+\frac{i\left(\hat{p}-p_{p}(t)\right)}{\sqrt{2 m \omega_{0} \hbar} R_{B}(t)}\right\}, \\
& \hat{A}_{0}^{\dagger}(t)=e^{-i \eta(t)}\left\{\left[\sqrt{\frac{m \omega_{0}}{2 \hbar}} R_{B}(t)-\frac{i \mu(t)}{\sqrt{2 m \omega_{0} \hbar} R_{B}(t)}\left(B(t)+\frac{\dot{R}_{B}(t)}{R_{B}(t)}\right)\right]\left(\hat{q}-x_{p}(t)\right)-\frac{i\left(\hat{p}-p_{p}(t)\right)}{\sqrt{2 m \omega_{0} \hbar} R_{B}(t)}\right\},
\end{aligned}
$$

where $\eta(t)$ is given by (26), and $\hat{I}_{0}(t)$ becomes

$$
\hat{I}_{0}(t)=\frac{m \omega_{0}}{2} R_{B}^{2}(t)\left(\hat{q}-x_{p}(t)\right)^{2}+\frac{1}{2 m \omega_{0} R_{B}^{2}(t)}\left[\left(\hat{p}-p_{p}(t)\right)+\mu(t)\left(B(t)+\frac{\dot{R}_{B}(t)}{R_{B}(t)}\right)\left(\hat{q}-x_{p}(t)\right)\right]^{2} .
$$


Then, by construction, we have $\hat{A}_{0}(t) \Phi_{\alpha}(q, t)=\alpha \Phi_{\alpha}(q, t)$, showing that coherent states $\Phi_{\alpha}(q, t)$ are eigenstates of $\hat{A}_{0}(t)$, corresponding to eigenvalues $\alpha=\alpha_{1}+i \alpha_{2}$. It is known that for a given Hamiltonian, the invariants depend on the initial wave functions. The invariant $\hat{I}_{0}(t)$ found here corresponds to the initial state $\varphi_{k}(q)$, and $\hat{I}_{0}(t)$ is special in the sense that its eigenstates are $\Psi_{k}(q, t)$ corresponding to the time-independent eigenvalues $E_{k}=\left(\hbar / \omega_{0}\right)(k+1 / 2)$ of the standard Hamiltonian $\hat{H}_{0}$, that is, $\hat{I}_{0}(t) \Psi_{k}(q, t)=E_{k} \Psi_{k}(q, t), k=0,1,2, \ldots$ On the other hand, if we define the operators

$$
\hat{A}(t)=e^{-i \eta(t)} \hat{A}_{0}(t), \quad \hat{A}^{\dagger}(t)=e^{i \eta(t)} \hat{A}_{0}^{\dagger}(t),
$$

then $\hat{I}(t)=\hbar\left(\hat{A}^{\dagger}(t) \hat{A}(t)+1 / 2\right)=\hat{I}_{0}(t)$, and $\hat{A}(t) \Phi_{\alpha}(q, t)=\alpha e^{-i \eta(t)} \Phi_{\alpha}(q, t)$, that is, coherent states are eigenstates of $\hat{A}(t)$, corresponding to eigenvalues $\alpha(t)=\alpha e^{-i \eta(t)}$. These relations show that the evolution operator approach agrees with the Lewis-Riesenfeld approach in Ref. 27, and results will be exactly same, when the invariant $\hat{I}(t)$ in Ref. 27 corresponds to the particular solution $\varrho(t)$ of the Eq. (30) with the specific initial conditions (31).

Now, using that $\langle\hat{q}\rangle_{\alpha}(t)=\left\langle\phi_{\alpha}\left(q, t_{0}\right)\left|\hat{q}_{H}(t)\right| \phi_{\alpha}\left(q, t_{0}\right)\right\rangle$, the expectation value of position at coherent state $\Phi_{\alpha}(q, t)$ is

$$
\langle\hat{q}\rangle_{\alpha}(t)=\sqrt{\frac{2 \hbar}{m \omega_{0}}}\left(\frac{\alpha_{1}}{x_{0}} x_{1}(t)+\alpha_{2}\left(m \omega_{0} x_{0}\right) x_{2}(t)\right)+x_{p}(t),
$$

and using that $\langle\hat{p}\rangle_{\alpha}(t)=\left\langle\phi_{\alpha}\left(q, t_{0}\right)\left|\hat{p}_{H}(t)\right| \phi_{\alpha}\left(q, t_{0}\right)\right\rangle$, the expectation value of the momentum is

$$
\langle\hat{p}\rangle_{\alpha}(t)=\sqrt{\frac{2 \hbar}{m \omega_{0}}}\left(\frac{\alpha_{1}}{x_{0}} p_{1}(t)+\alpha_{2}\left(m \omega_{0} x_{0}\right) p_{2}(t)\right)+p_{p}(t),
$$

where $p_{1}(t)=\mu(t)\left(\dot{x}_{1}(t)-B(t) x_{1}(t)\right)$ and $p_{2}(t)=\mu(t)\left(\dot{x}_{2}(t)-B(t) x_{2}(t)\right)$ are two linearly independent solutions of the homogeneous part of the classical oscillator (5) in momentum space. Obviously, the expectation values at coherent states satisfy the classical equations of motion. We note that the homogeneous solutions of the classical oscillator can be written also in terms of the functions $\varrho(t)$ and $\eta(t)$ as

$$
x_{1}(t)=\sqrt{m \omega_{0}} x_{0} \varrho(t) \cos \eta(t), \quad x_{2}(t)=-\frac{1}{\sqrt{m \omega_{0}} x_{0}} \varrho(t) \sin \eta(t) .
$$

Then, it is not difficult to see that, if the expectation values $\langle\hat{q}\rangle_{\alpha}(t)$ and $\langle\hat{p}\rangle_{\alpha}(t)$ are evaluated using the operators $\hat{A}(t)$ and $\hat{A}^{\dagger}(t)$, as in the LR-invariant approach, ${ }^{27}$ they will agree with (40) and (41). Next, finding the expectation of squares

$$
\begin{aligned}
\left\langle\hat{q}^{2}\right\rangle_{\alpha}(t)= & {\left[\sqrt{\frac{2 \hbar}{m \omega_{0}}}\left(\alpha_{1} \frac{x_{1}(t)}{x_{0}}+\alpha_{2}\left(m \omega_{0} x_{0}\right) x_{2}(t)\right)+x_{p}(t)\right]^{2}+\frac{\hbar}{2 m \omega_{0} R_{B}^{2}(t)}, } \\
\left\langle\hat{p}^{2}\right\rangle_{\alpha}(t)= & {\left[\sqrt{\frac{2 \hbar}{m \omega_{0}}}\left(\frac{\alpha_{1}}{x_{0}} p_{1}(t)+\alpha_{2}\left(m \omega_{0} x_{0}\right) p_{2}(t)\right)+p_{p}(t)\right]^{2} } \\
& +\frac{\hbar}{2 m \omega_{0}}\left[\left(m \omega_{0} R_{B}(t)\right)^{2}+\frac{\mu^{2}(t)}{R_{B}^{2}(t)}\left(\frac{\dot{R}_{B}(t)}{R_{B}(t)}+B(t)\right)^{2}\right]
\end{aligned}
$$

gives the fluctuations for $\hat{q}$ and $\hat{p}$,

$$
\begin{aligned}
(\Delta \hat{q})_{\alpha}(t) & =\sqrt{\frac{\hbar}{2 m \omega_{0}}} \frac{1}{R_{B}(t)}, \\
(\Delta \hat{p})_{\alpha}(t) & =\sqrt{\frac{m \omega_{0} \hbar}{2}} R_{B}(t) \sqrt{1+\frac{\mu^{2}(t)}{\left(m \omega_{0} R_{B}^{2}(t)\right)^{2}}\left(\frac{\dot{R}_{B}(t)}{R_{B}(t)}+B(t)\right)^{2}} .
\end{aligned}
$$

We see that the expectation values depend on all parameters of the Hamiltonian; however, the fluctuations depend only on $\mu(t), \omega^{2}(t)$, and parameter $B(t)$. In other words, uncertainties does not 
depend on the external linear terms, which contribute only to displacement of the wave packet. Finally, the uncertainty relation for the generalized harmonic oscillator with time dependent parameters is

$$
(\Delta \hat{q})_{\alpha}(\Delta \hat{p})_{\alpha}(t)=\frac{\hbar}{2} \sqrt{1+\frac{\mu^{2}(t)}{\left(m \omega_{0} R_{B}^{2}(t)\right)^{2}}\left(\frac{\dot{R}_{B}(t)}{R_{B}(t)}+B(t)\right)^{2}},
$$

where clearly $(\Delta \hat{q})_{\alpha}(\Delta \hat{p})_{\alpha} \geq \hbar / 2$, and this relation coincides with the uncertainty relation at Gaussian (ground) state for $k=0$ in Eq. (34).

As a result we can say that our formulas confirm the well-known properties, such as coherent states of the generalized parametric oscillator are displaced Gaussian wave packets, they are eigenstates of the annihilation operator of a dynamical invariant, and follow the classical trajectory. However, they are spreading or squeezing in time, since $(\Delta \hat{q})_{\alpha}(t)$ given by (43) depends explicitly on time, and are no longer minimum uncertainty states, as we can see from Eq. (45).

\section{EXACTLY SOLVABLE MODELS}

Since the solution of the generalized time-dependent quadratic oscillator is completely determined by the corresponding classical equation of motion, it is interesting to consider cases for which this equation has exact closed form solutions. Here, we introduce generalized oscillator models related with the classical orthogonal polynomials, which are eigenfunctions of certain singular Sturm-Liouville problems, and are also solutions of a classical oscillator of the form

$$
\ddot{x}+\frac{\dot{\mu}(t)}{\mu(t)} \dot{x}+\Omega^{2}(t) x=0 .
$$

Precisely, we shall consider problems in which the damping $\Gamma(t)=\dot{\mu}(t) / \mu(t)$ and the modified frequency

$$
\Omega^{2}(t)=\omega^{2}(t)-\left(\dot{B}+B^{2}+\frac{\dot{\mu}}{\mu} B\right)
$$

are coefficients of the classical Hermite, Laguerre, and Jacobi differential equations. Clearly, this requires a special relation between the original frequency $\omega^{2}(t)$ and the parameter $B(t)$. To see this relation, we denote by

$$
\Lambda^{2}(t)=-\left(\dot{B}+B^{2}+\frac{\dot{\mu}}{\mu} B\right)
$$

the modification of the original frequency $\omega^{2}(t)$. Then, substitution $B(t)=\dot{y} / y$ in (47) gives differential equation for classical oscillator with frequency $\Lambda^{2}(t)$,

$$
\ddot{y}+\frac{\dot{\mu}}{\mu} \dot{y}+\Lambda^{2}(t) y=0 .
$$

This suggests that it is possible to obtain the exact solutions of the classical oscillator (46), when for given parameters $\mu(t), \omega^{2}(t)$, and $B(t)$ Equations (46) and (48) are related with the same Sturm-Liouville problem, that is, the frequencies $\Omega^{2}(t)$ and $\Lambda^{2}(t)$ are compatible. According to this, in Subsections III A-III C we introduce generalized Hermite, Laguerre, and Jacobi type oscillators.

\section{A. Hermite type generalized quantum oscillator}

We define the Hermite type generalized quantum oscillator by the Hamiltonian

$$
\hat{H}_{g}(t)=\frac{e^{t^{2}}}{2} \hat{p}^{2}+n e^{-t^{2}} \hat{q}^{2}+\left(\frac{\dot{H}_{r}(t)}{H_{r}(t)}\right) \frac{(\hat{q} \hat{p}+\hat{p} \hat{q})}{2}+D(t) \hat{q}+E(t) \hat{p}+F(t)
$$

with variable mass $\mu(t)=e^{-t^{2}}$, constant frequency $\omega^{2}(t)=2 n, n=0,1,2, \ldots$, and mixed term parameter 
$B(t)=\dot{H}_{r} / H_{r}$, where

$$
H_{r}(t)=r ! \sum_{k=0}^{\lfloor r / 2\rfloor} \frac{(-1)^{k}}{k !(r-2 k) !}(2 t)^{r-2 k}, r=0,1,2, \ldots
$$

are the standard Hermite polynomials, and external parameters $D(t), E(t)$, and $F(t)$. Then, the classical equation of motion is a forced Hermite differential equation

$$
\ddot{x}-2 t \dot{x}+2(n+r) x=-\frac{1}{\mu} D+\dot{E}+\left(\frac{\dot{\mu}}{\mu}+\frac{\dot{H}_{r}}{H_{r}}\right) E, \quad-\infty<t<\infty,
$$

with time-variable damping $\Gamma(t)=\dot{\mu} / \mu=-2 t$, and modified frequency $\Omega^{2}(t)=\omega^{2}(t)+\Lambda^{2}(t)=$ $2(n+r)$, where $r=0$ corresponds to the case $B(t)=0$. Note that coefficients of the homogeneous equation are continuous, despite that $B(t)$ has singularities at the zeros of $H_{r}(t)$. Then, essential properties of the particular solution will depend on the total forcing term in Eq. (50). By special choice of $E(t)$, it is possible to remove the singularities in the total force, so that it also becomes continuous. Then, solution of the quantum oscillator with Hamiltonian (49) can be written in terms of two independent homogeneous solutions $x_{1}(t)$ and $x_{2}(t)$ of (50), satisfying the initial conditions

$$
\begin{gathered}
x_{1}\left(t_{0}\right)=x_{0} \neq 0, \quad \dot{x}_{1}\left(t_{0}\right)=x_{0} \dot{H}_{r}\left(t_{0}\right) / H_{r}\left(t_{0}\right), \quad H_{r}\left(t_{0}\right) \neq 0, \\
x_{2}\left(t_{0}\right)=0, \quad \dot{x}_{2}\left(t_{0}\right)=1 / \mu\left(t_{0}\right) x_{0},
\end{gathered}
$$

respectively, and a particular solution $x_{p}(t)$ of (50) satisfying $x_{p}\left(t_{0}\right)=0, \dot{x}_{p}\left(t_{0}\right)=E\left(t_{0}\right)$. When these solutions are smooth, probability densities of the wave functions and the coherent states (38) will be also smooth. However, singularities of $B(t)$ will be reflected in momentum expectation values (41) and fluctuations (44), and in the uncertainty relation (45), as we will show in the examples. Before this, we recall that the solution of the homogeneous Eq. (50) with given initial conditions, in general, will be a linear combination of Hermite polynomial and a confluent hypergeometric function of first kind ${ }_{1} F_{1}(a, b ; t)$, which is represented by the series

$$
{ }_{1} F_{1}(a, b ; t)=\sum_{n=0}^{\infty} \frac{(a)_{n}}{(b)_{n}} \frac{t^{n}}{n !}, \quad b \neq 0,-1,-2, \ldots,
$$

where $(a)_{n}$ and $(b)_{n}$ are Pochhammer symbols that are given by the relation $(a)_{n}=\Gamma(a+n) / \Gamma(a)$. However, there are some cases which can be easily treated:

(i) when $n$ is an odd positive integer and $r$ is an even positive integer, that is, $n=2 k+1$ and $r=2 s, k, s=0,1,2,3, \ldots, t_{0}=0$, and $x_{0}=1 / \dot{H}_{2(k+s)+1}(0), x_{1}(t)=x_{0}\left({ }_{1} F_{1}(-(2(k+s)+\right.$ $\left.1) / 2,1 / 2 ; t^{2}\right)$ ) and second solution is the Hermite polynomial $x_{2}(t)=H_{2(k+s)+1}(t)$;

(ii) when $n$ and $r$ are both positive even integers, that is, $n=2 k$ and $r=2 s, k, s=0,1,2,3, \ldots$, $t_{0}=0$, and $x_{0}=H_{2(k+s)}(0)$, the first solution is the Hermite polynomial, $x_{1}(t)=H_{2(k+s)}(t)$, and the second linearly independent solution is $x_{2}(t)=t / x_{0}\left({ }_{1} F_{1}\left(-(k+s-1 / 2), 3 / 2 ; t^{2}\right)\right)$.

On the other hand, the particular solution of Eq. (50) will depend on the choice of the external parameters. We write some special cases which could be of interest:

(a) When $B(t)=\dot{H}_{r} / H_{r}, D(t)=\left[(d / d t)\left(e^{-t^{2}} H_{r} E(t)\right)\right] / H_{r}(t)$, and $E\left(t_{0}\right)=0$, the total force in (50) is zero, so that $x_{p}(t)=0$, and $p_{p}(t)=-\mu(t) E(t)$.

(b) When $B(t)=0$ and $D(t)=-\mu(t) \omega^{2}(t) \int_{t_{0}}^{t} E\left(t^{\prime}\right) d t^{\prime}, x_{p}(t)=\int_{t_{0}}^{t} E\left(t^{\prime}\right) d t^{\prime}$ and $p_{p}(t)=0$.

(c) When $B(t)=\dot{H}_{r} / H_{r}, D(t) \neq 0, E(t)=0$,

$$
x_{p}(t)=-x_{1}(t) \int^{t} \frac{1}{\mu(s) x_{1}^{2}(s)} \int_{t_{0}}^{s} D(\xi) x_{1}(\xi) d \xi d s, x_{p}\left(t_{0}\right)=0 .
$$

To get better insight into the problem, we give concrete examples. 
Example 1. (a) Let $n=2, r=2$, and $B(t)=\dot{H}_{2}(t) / H_{2}(t)$, but $D(t)=E(t)=F(t)=0$, so that there is no linear force. Then, the Hamiltonian is

$$
\hat{H}_{g}(t)=\frac{e^{t^{2}}}{2} \hat{p}^{2}+2 e^{-t^{2}} \hat{q}^{2}+\left(\frac{\dot{H}_{2}(t)}{H_{2}(t)}\right) \frac{(\hat{q} \hat{p}+\hat{p} \hat{q})}{2},
$$

and the corresponding classical equation of motion becomes $\ddot{x}-2 t \dot{x}+8 x=0$. For $t_{0}=0$, two linearly independent homogeneous solutions, satisfying the initial conditions $x_{1}(0)=12, \dot{x}_{1}(0)=0$, and $x_{2}(0)=0, \dot{x}_{2}(0)=1 / 12$, are

$$
x_{1}(t)=H_{4}(t), \quad x_{2}(t)=\frac{t}{12}{ }_{1} F_{1}\left(\frac{-3}{2}, \frac{3}{2} ; t^{2}\right)=\frac{1}{192}\left[e^{t^{2}}\left(\frac{-H_{3}(t)}{2}+2 H_{1}(t)\right)+\frac{\sqrt{\pi}}{4} H_{4}(t) \operatorname{erfi}(t)\right],
$$

where erfi $(t)$ is the imaginary error function defined by erfi $(t)=(2 / \sqrt{\pi}) \int_{0}^{t} e^{s^{2}} d s$, and

$$
R_{B}(t)=\sqrt{\frac{144}{H_{4}^{2}(t)+\left(12 m \omega_{0} t\left({ }_{1} F_{1}\left(\frac{-3}{2}, \frac{3}{2} ; t^{2}\right)\right)\right)^{2}}},
$$

which is smooth, oscillatory in a finite time-interval near $t=0$, and $R_{B}(t) \rightarrow 0$ as $t \rightarrow \infty$. Then, the probability density $\rho_{k}^{n, r}(q, t)=\left|\Psi_{k}^{n, r}(q, t)\right|^{2}$ for $n=2, r=2$ becomes

$$
\rho_{k}^{2,2}(q, t)=N_{k}^{2} R_{B}(t) \exp \left(-\frac{m \omega_{0}}{\hbar} R_{B}^{2}(t) q^{2}\right) H_{k}^{2}\left(\sqrt{\frac{m \omega_{0}}{\hbar}} R_{B}(t) q\right),
$$

and in Fig. 1(a) we plot it for $k=2$, where one can see that it is smooth, and since $k=2$, it has two moving zeros. Also, the essentially nontrivial localization of the particle takes place for $|t| \leq 2$, and for $|t| \geq 2$ the probability density spreads along q-coordinate. The probability density in coherent state $\rho_{\alpha}^{n, r}(q, t)=\left|\Phi_{\alpha}^{n, r}(q, t)\right|^{2}$ for $n=2, r=2$ is

$$
\rho_{\alpha}^{n, r}(q, t)=\sqrt{\frac{m \omega_{0}}{\pi \hbar}} R_{B}(t) \exp \left[-\left(\frac{m \omega_{0}}{\hbar}\right) R_{B}^{2}(t)\left(q-\langle\hat{q}\rangle_{\alpha}(t)\right)^{2}\right]
$$

and

$$
\begin{aligned}
\langle\hat{q}\rangle_{\alpha}(t)= & \sqrt{\frac{2 \hbar}{m \omega_{0}}}\left[\alpha_{1} \frac{H_{4}(t)}{12}+\alpha_{2}\left(m \omega_{0} t\right)\left({ }_{1} F_{1}\left(\frac{-3}{2}, \frac{3}{2} ; t^{2}\right)\right)\right], \\
\langle\hat{p}\rangle_{\alpha}(t)= & \sqrt{\frac{2 \hbar}{m \omega_{0}}} e^{-t^{2}}\left\{\frac{\alpha_{1}}{12}\left(\dot{H}_{4}-\frac{\dot{H}_{2}}{H_{2}} H_{4}(t)\right)\right. \\
& \left.+\alpha_{2}\left(12 m \omega_{0}\right)\left[\left(1-t \frac{\dot{H}_{2}(t)}{H_{2}(t)}\right)\left({ }_{1} F_{1}\left(\frac{-3}{2}, \frac{3}{2} ; t^{2}\right)\right)-t\left({ }_{1} F_{1}\left(\frac{-1}{2}, \frac{5}{2} ; t^{2}\right)\right)\right]\right\} .
\end{aligned}
$$

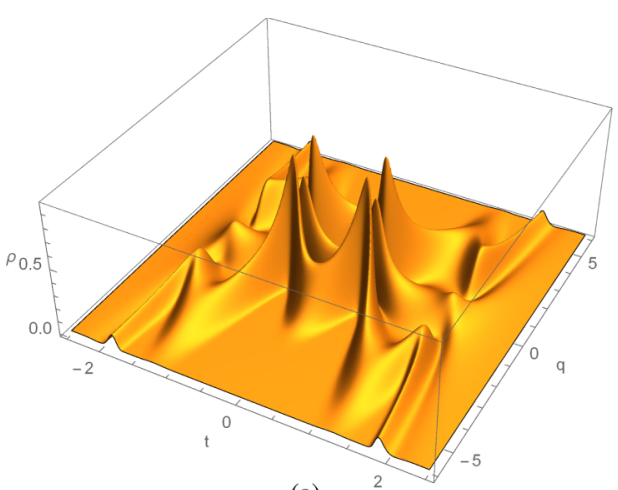

(a)

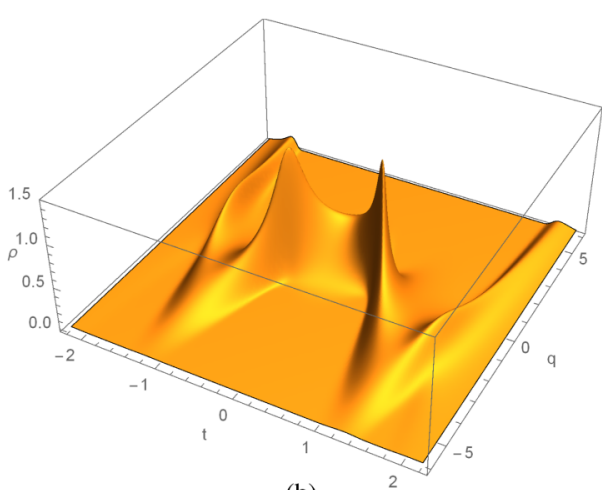

(b)

FIG. 1. Hermite type generalized oscillator, when $D(t)=E(t)=F(t)=0$. (a) Probability density $\rho_{2}^{2,2}(q, t)=\left|\Psi_{2}^{2,2}(q, t)\right|^{2}$, $n=r=k=2$. (b) Probability density in coherent state $\rho_{\alpha}^{2,2}(q, t)=\left|\Phi_{\alpha}^{2,2}(q, t)\right|^{2}$ for $\alpha=1 / \sqrt{2}+i(1 / \sqrt{2}), n=r=2$. 
In Fig. 1(b) we plot (52) for $\alpha=1 / \sqrt{2}+i(1 / \sqrt{2})$. We observe that it is a Gaussian type wave packet following the classical trajectory.

With $R_{B}(t)$ as found in (51), fluctuations for $\hat{q}$ and $\hat{p}$ and uncertainty relation at coherent states take the form

$$
\begin{aligned}
(\Delta \hat{q})_{\alpha}(t) & =\sqrt{\frac{\hbar}{2 m \omega_{0}}} \frac{1}{R_{B}(t)}, \\
(\Delta \hat{p})_{\alpha}(t) & =\sqrt{\frac{m \omega_{0} \hbar}{2}} R_{B}(t) \sqrt{\left(1+\frac{e^{-2 t^{2}}}{\left(m \omega_{0} R_{B}^{2}(t)\right)^{2}}\left(\frac{\dot{R}_{B}(t)}{R_{B}(t)}+\frac{\dot{H}_{2}(t)}{H_{2}(t)}\right)^{2}\right)}, \\
(\Delta \hat{q})_{\alpha}(\Delta \hat{p})_{\alpha}(t) & =\frac{\hbar}{2} \sqrt{1+\frac{e^{-2 t^{2}}}{\left(m \omega_{0} R_{B}^{2}(t)\right)^{2}}\left(\frac{\dot{R}_{B}(t)}{R_{B}(t)}+\frac{\dot{H}_{2}(t)}{H_{2}(t)}\right)^{2}} .
\end{aligned}
$$

Since coefficients of the classical equation are continuous, the expectations (53) and fluctuations (55) of the position are smooth. In a finite time interval near the origin, $(\Delta \hat{q})_{\alpha}(t)$ oscillates, and for $|t| \rightarrow \infty$ we have increasing $(\Delta \hat{q})_{\alpha}(t)$, showing spreading in position. On the other hand, the singularities of the coefficient $B(t)$ at zeros of $H_{2}(t)$, are reflected in the expectations (54) and fluctuations (56) of the momentum. Then, as shown in Fig. 2, the uncertainty (57) is oscillatory in a finite time interval near the origin, but it has singularities at the two zeros of the Hermite polynomial $H_{2}(t)$. As $|t| \rightarrow \infty,(\Delta \hat{q})_{\alpha}(\Delta \hat{p})_{\alpha}(t) \rightarrow \infty$, which shows that the uncertainties do not compensate each other in the limiting case.

(b) Now, we consider the oscillator in part (a) under the influence of linear external terms. That is, $n=2, r=2$, and we choose $D(t)=t H_{2}(t), E(t)=-\left(\left(1+2 t^{2}\right) H_{2}(t) e^{t^{2}}\right) / 4, F(t)=0$. Then, the Hamiltonian becomes

$$
H_{g}(t)=\frac{e^{t^{2}}}{2} \hat{p}^{2}+2 e^{-t^{2}} \hat{q}^{2}+\left(\frac{\dot{H}_{2}(t)}{H_{2}(t)}\right) \frac{(\hat{q} \hat{p}+\hat{p} \hat{q})}{2}+t H_{2}(t) \hat{q}-\frac{1}{4}\left(1+2 t^{2}\right) H_{2}(t) e^{t^{2}} \hat{p},
$$

and the corresponding classical equation is

$$
\ddot{x}-2 t \dot{x}+8 x=-\left(2 t H_{2}(t)+\frac{1}{2}\left(1+2 t^{2}\right) \dot{H}_{2}(t)\right) e^{t^{2}} .
$$

We note that, by above choice of $D(t)$ and $E(t), p_{p}(t)$ is zero and $E(t)$ compensates the singularities coming from $B(t)$, so that the forcing term in Eq. (58) is continuous. For $t_{0}=0$, two homogeneous solutions $x_{1}(t)$ and $x_{2}(t)$ of (58) are as given in part (a), and the particular solution satisfying

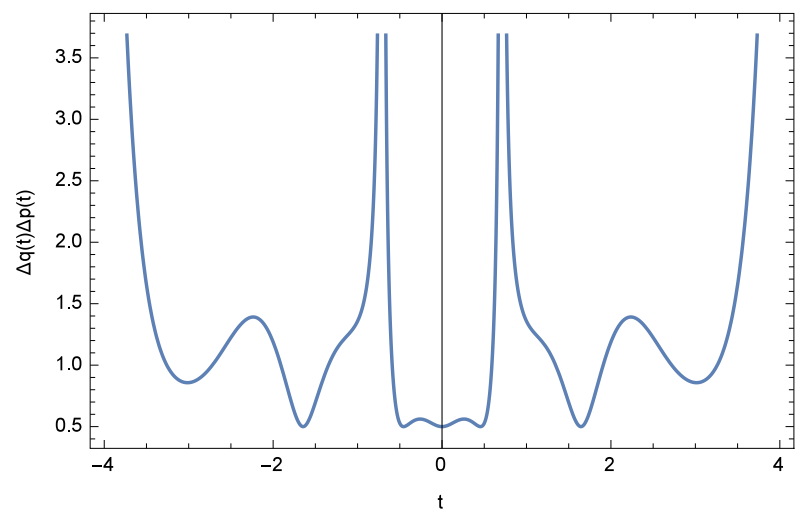

FIG. 2. Uncertainty relation for generalized Hermite oscillator, $n=r=2$. 


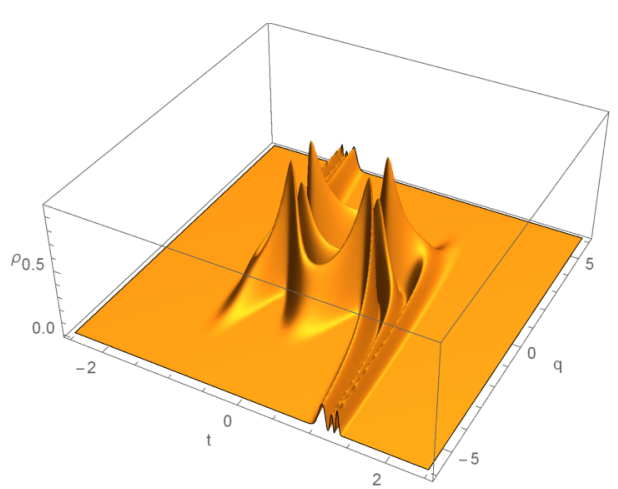

(a)

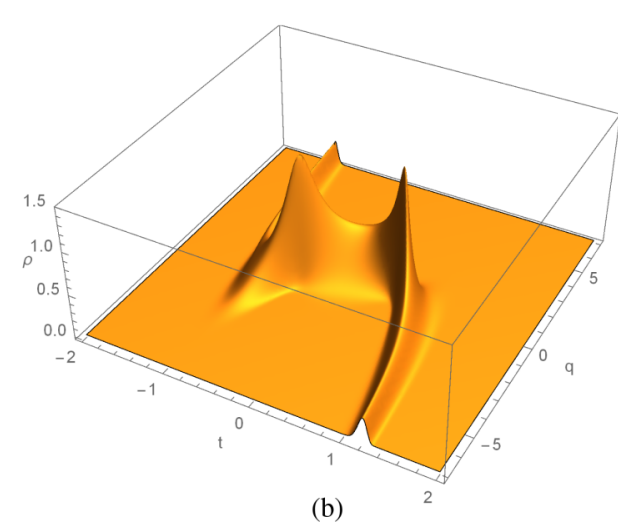

(b)

FIG. 3. Hermite type generalized oscillator, when $D(t)=t H_{2}(t), E(t)=-\left(\left(1+2 t^{2}\right) H_{2}(t) e^{t^{2}}\right) / 4, F(t)=0$. (a) Probability density $\rho_{2}^{2,2}(q, t)=\left|\Psi_{2}^{2,2}(q, t)\right|^{2}, n=r=k=2$. (b) Probability density in coherent states $\rho_{\alpha}^{2,2}(q, t)=\left|\Phi_{\alpha}^{2,2}(q, t)\right|^{2}$ for $\alpha=$ $1 / \sqrt{2}+i(1 / \sqrt{2}), n=r=2$.

$x_{p}(0)=0, \dot{x}_{p}(0)=1 / 2$, is $x_{p}(t)=-\left(t H_{2}(t) e^{t^{2}}\right) / 4$. This gives new probability density

$$
\begin{aligned}
\rho_{k}^{2,2}(q, t)= & N_{k}^{2} R_{B}(t) \exp \left(-\left(\sqrt{\frac{m \omega_{0}}{\hbar}} R_{B}(t)\left(q+\frac{1}{4} t H_{2}(t) e^{t^{2}}\right)\right)^{2}\right) \\
& \times H_{k}^{2}\left(\sqrt{\frac{m \omega_{0}}{\hbar}} R_{B}(t)\left(q+\frac{1}{4} t H_{2}(t) e^{t^{2}}\right)\right),
\end{aligned}
$$

with $R_{B}(t)$ as found in part (a), but position coordinate displaced by $x_{p}(t)$. The influence of this displacement can be seen in Fig. 3(a). On the other hand, the new probability density in coherent states is in the form of the Eq. (52) with

$$
\langle\hat{q}\rangle_{\alpha}(t)=\sqrt{\frac{2 \hbar}{m \omega_{0}}}\left[\alpha_{1} \frac{H_{4}(t)}{12}+\alpha_{2}\left(m \omega_{0} t\right)\left({ }_{1} F_{1}\left(\frac{-3}{2}, \frac{3}{2} ; t^{2}\right)\right)\right]-\frac{1}{4} t H_{2}(t) e^{t^{2}},
$$

and its evolution is shown in Fig. 3(b). Clearly, expectation of position (53) is also displaced by $x_{p}(t)$, but the expectation of momentum $\langle\hat{p}\rangle_{\alpha}(t)$ given by Eq. (54) does not change, since in this example $p_{p}(t)=0$. From the general results we know that the fluctuations and uncertainty relation obtained in part (a) does not change under the influence of the linear external terms.

\section{B. Associated Laguerre type generalized quantum oscillator}

We define a generalized associated Laguerre type oscillator by the Hamiltonian

$$
\hat{H}_{g}(t)=\frac{e^{t}}{2 t^{m+1}} \hat{p}^{2}+\frac{n t^{m}}{2 e^{t}} \hat{q}^{2}+\left(\frac{\dot{L}_{r}^{m}(t)}{L_{r}^{m}(t)}\right) \frac{(\hat{q} \hat{p}+\hat{p} \hat{q})}{2}+D(t) \hat{q}+E(t) \hat{p}+F(t),
$$

with variable mass $\mu(t)=t^{m+1} e^{-t}, m>-1$, variable frequency $\omega^{2}(t)=n / t, n=0,1,2, \ldots$, and $B(t)=\dot{L}_{r}^{m}(t) / L_{r}^{m}(t), r=0,1,2, \ldots$, where $L_{r}^{m}(t)=e^{t} t^{-m}(r !)^{-1} d^{r}\left(e^{-t} t^{r+m}\right) / d t^{r}$ are the associated Laguerre polynomials. The corresponding classical oscillator is a forced associated Laguerre differential equation

$$
\ddot{x}+\frac{(m+1-t)}{t} \dot{x}+\frac{(n+r)}{t} x=-\frac{e^{t}}{t^{m+1}} D+\dot{E}+\left(\frac{m+1-t}{t}+\frac{\dot{L}_{r}^{m}}{L_{r}^{m}}\right) E, \quad 0<t<\infty,
$$

with damping $\Gamma(t)=(m+1-t) / t$, and modified frequency $\Omega^{2}(t)=(n+r) / t$. Here, we shall examine and give example for the case when $m=0$. 


\section{Laguerre type generalized quantum oscillator}

For $\mathrm{m}=0$, the Hamiltonian for a Laguerre type generalized oscillator is

$$
\hat{H}_{g}(t)=\frac{e^{t}}{2 t} \hat{p}^{2}+\frac{n}{2 e^{t}} \hat{q}^{2}+\left(\frac{\dot{L}_{r}(t)}{L_{r}(t)}\right) \frac{(\hat{q} \hat{p}+\hat{p} \hat{q})}{2}+D(t) \hat{q}+E(t) \hat{p}+F(t),
$$

where $\mu(t)=t e^{-t}, \omega^{2}(t)=n / t, n=0,1,2, \ldots, t \in(0, \infty), B(t)=\dot{L}_{r}(t) / L_{r}(t)$, and

$$
L_{r}(t)=\sum_{k=0}^{r}\left(\begin{array}{l}
r \\
k
\end{array}\right) \frac{(-1)^{k}}{k !} t^{k}, r=0,1,2, \ldots
$$

are the standard Laguerre polynomials. Then, the corresponding classical oscillator is a forced Laguerre differential equation

$$
\ddot{x}+\frac{(1-t)}{t} \dot{x}+\frac{(n+r)}{t} x=-\frac{e^{t}}{t} D+\dot{E}+\left(\frac{1-t}{t}+\frac{\dot{L}_{r}}{L_{r}}\right) E, \quad 0<t<\infty,
$$

with $\Gamma(t)=(1-t) / t$, and $\Omega^{2}(t)=(n+r) / t$. Since coefficients of the homogeneous equation are continuous for $t>0$, assuming the total force is also continuous for $t>0$, the solution of the quantum oscillator with Hamiltonian (61) can be written in terms of two independent homogeneous solutions $x_{1}(t)$ and $x_{2}(t)$ of (62), satisfying the initial conditions

$$
\begin{gathered}
x_{1}\left(t_{0}\right)=x_{0} \neq 0, \quad \dot{x}_{1}\left(t_{0}\right)=x_{0} \frac{\dot{L}_{r}\left(t_{0}\right)}{L_{r}\left(t_{0}\right)}, \quad L_{r}\left(t_{0}\right) \neq 0, \\
x_{2}\left(t_{0}\right)=0, \quad \dot{x}_{2}\left(t_{0}\right)=1 / \mu\left(t_{0}\right) x_{0},
\end{gathered}
$$

respectively, and a particular solution $x_{p}(t)$ of (62) satisfying $x_{p}\left(t_{0}\right)=0, \dot{x}_{p}\left(t_{0}\right)=E\left(t_{0}\right)$.

Example 2. (a) Let $n=r=1, B(t)=\dot{L}_{1}(t) / L_{1}(t)$ and $D(t)=E(t)=F(t)=0$. Then, the Hamiltonian becomes

$$
\hat{H}_{g}(t)=\frac{e^{t}}{2 t} \hat{p}^{2}+\frac{1}{2 e^{t}} \hat{q}^{2}+\left(\frac{\dot{L}_{1}(t)}{L_{1}(t)}\right) \frac{(\hat{q} \hat{p}+\hat{p} \hat{q})}{2},
$$

and the classical equation is $\ddot{x}+(1-t) / t \dot{x}+2 / t x=0$. For $t_{0}=2$, two solutions satisfying the conditions $x_{1}(2)=1, \dot{x}_{1}(2)=1$ and $x_{2}(2)=0, \dot{x}(2)=e^{2} / 2$, respectively, are

$$
x_{1}(t)=\frac{1}{e^{2}}\left[e^{t}(t-3)-2 L_{2}(t)\left(e^{2}-E i(2)+E i(t)\right)\right]
$$

and

$$
x_{2}(t)=\frac{1}{2}\left[e^{t}(t-3)-L_{2}(t)\left(e^{2}-2 E i(2)+2 E i(t)\right)\right],
$$

where $E i(t)$ is the exponential integral defined by $E i(t)=-\int_{-t}^{\infty}\left(e^{-s} / s\right) d s$. With above $x_{1}(t)$ and $x_{2}(t)$, we have

$$
R_{B}(t)=\sqrt{\frac{1}{x_{1}^{2}(t)+\left(m \omega_{0} x_{2}(t)\right)^{2}}},
$$

which is smooth for $t>0$ and $R_{B}(t) \rightarrow 0$ as $t \rightarrow \infty$. Then, the corresponding probability density for $n=1, r=1$ is

$$
\rho_{k}^{1,1}(q, t)=N_{k}^{2} R_{B}(t) \exp \left(-\frac{m \omega_{0}}{\hbar} R_{B}^{2}(t) q^{2}\right) H_{k}^{2}\left(\sqrt{\frac{m \omega_{0}}{\hbar}} R_{B}(t) q\right),
$$

and in Fig. 4(a) we plot it for $k=2$. The probability density is a smooth function, which has two moving zeros, since $k=2$. It shows oscillatory behavior in a finite time interval near $t=0$, and then spreads along the $q$-coordinate. The probability density $\rho_{\alpha}^{1,1}(q, t)$ has the form of Eq. (52) and the 


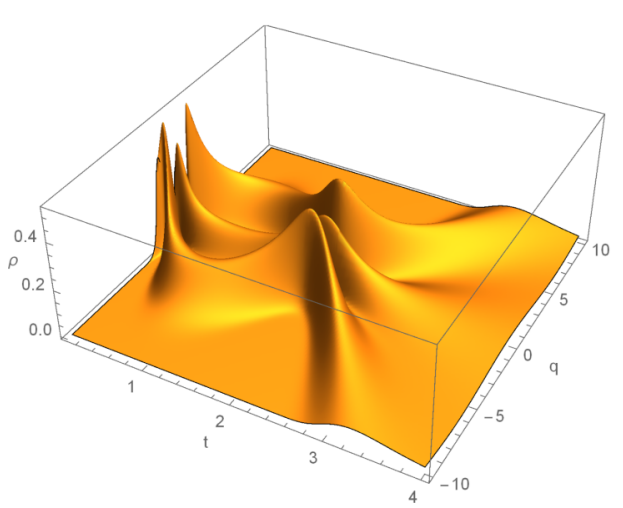

(a)

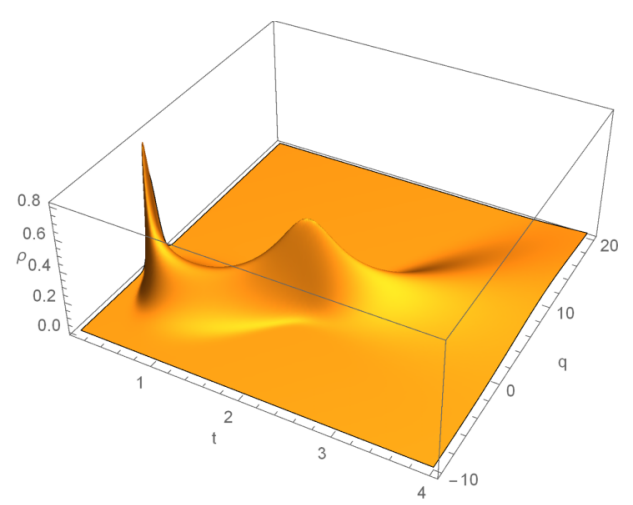

(b)

FIG. 4. Laguerre type generalized oscillator, when $D(t)=E(t)=F(t)=0$. (a) Probability density $\rho_{2}^{1,1}(q, t)=\left|\Psi_{2}^{1,1}(q, t)\right|^{2}$, $n=r=1$, and $k=2$. (b) Probability density $\rho_{\alpha}^{1,1}(q, t)=\left|\Psi_{\alpha}^{1,1}(q, t)\right|^{2}$ in coherent states for $\alpha=1 / \sqrt{2}+i(1 / \sqrt{2}), n=r=1$.

expectation values are

$$
\begin{gathered}
\langle\hat{q}\rangle_{\alpha}(t)=\sqrt{\frac{2 \hbar}{m \omega_{0}}}\left(\alpha_{1} x_{1}(t)+\alpha_{2}\left(m \omega_{0}\right) x_{2}(t)\right), \\
\langle\hat{p}\rangle_{\alpha}(t)=\sqrt{\frac{2 \hbar}{m \omega_{0}}} t e^{-t}\left[\alpha_{1}\left(\dot{x}_{1}(t)-\frac{\dot{L}_{1}(t)}{L_{1}(t)} x_{1}(t)\right)+\alpha_{2}\left(m \omega_{0}\right)\left(\dot{x}_{2}(t)-\frac{\dot{L}_{1}(t)}{L_{1}(t)} x_{2}(t)\right)\right],
\end{gathered}
$$

where $x_{1}(t), x_{2}(t)$ are defined by (63) and (64). In Fig. 4(b) we plot $\rho_{\alpha}^{1,1}(q, t)$. Also, (66) and (67) show that the wave packet of the coherent state follows the trajectory of the classical particle. With $R_{B}(t)$ calculated from (65), the fluctuations and uncertainty relation becomes

$$
\begin{aligned}
(\Delta \hat{q})_{\alpha}(t) & =\sqrt{\frac{\hbar}{2 m \omega_{0}}} \frac{1}{R_{B}(t)}, \\
(\Delta \hat{p})_{\alpha}(t) & =\sqrt{\frac{m \omega_{0} \hbar}{2}} R_{B}(t) \sqrt{1+\frac{t^{2} e^{-2 t}}{\left(m \omega_{0} R_{B}^{2}(t)\right)^{2}}\left(\frac{\dot{R}_{B}(t)}{R_{B}(t)}+\frac{\dot{L}_{1}(t)}{L_{1}(t)}\right)^{2}}, \\
(\Delta \hat{q})_{\alpha}(\Delta \hat{p})_{\alpha} & =\frac{\hbar}{2} \sqrt{1+\frac{t^{2} e^{-2 t}}{\left(m \omega_{0} R_{B}^{2}(t)\right)^{2}}\left(\frac{\dot{R}_{B}(t)}{R_{B}(t)}+\frac{\dot{L}_{1}(t)}{L_{1}(t)}\right)^{2}} .
\end{aligned}
$$

Since the solution of the classical oscillator is given in terms of Laguerre polynomials and exponential functions, $(\Delta \hat{q})_{\alpha}(t)$ shows oscillatory behavior in a finite time interval near $t=0$, while for $|t| \rightarrow \infty,(\Delta \hat{q})_{\alpha}(t)$ goes to infinity, which confirms spreading in position coordinate. However, the singularity in parameter $B(t)$ at the zero of $L_{1}(t)$ appears both in the expectation (67) and fluctuation (69) of the momentum, where it becomes undefined. Consequently, the uncertainty relation also has singularity at finite time, where $L_{1}(t)=0$, and for $t \rightarrow 0$ and $t \rightarrow \infty$, one has $(\Delta \hat{q})_{\alpha}(\Delta \hat{p})_{\alpha} \rightarrow \infty$, as one can see in Fig. 5.

(b) Now, we consider the system in part (a) under the influence of linear external terms. That is, let $n=1, r=1$, and $D(t)=(t-2) L_{2}(t), E(t)=(1-t) L_{2}(t) e^{t}, F(t)=0$. Then, the corresponding Hamiltonian is

$$
\hat{H}_{g}(t)=\frac{e^{t}}{2 t} \hat{p}^{2}+\frac{1}{2 e^{t}} \hat{q}^{2}+\left(\frac{\dot{L}_{1}(t)}{L_{1}(t)}\right) \frac{(\hat{q} \hat{p}+\hat{p} \hat{q})}{2}+(t-2) L_{2}(t) \hat{q}+(1-t) L_{2}(t) e^{t} \hat{p},
$$

and the classical equation becomes

$$
\ddot{x}+\frac{1-t}{t} \dot{x}+\frac{2}{t} x=\frac{e^{t}}{t}\left(L_{1}^{2}(t)-1\right)+3 e^{t} L_{1}(t) \dot{L}_{1}(t), \quad 0<t<\infty .
$$




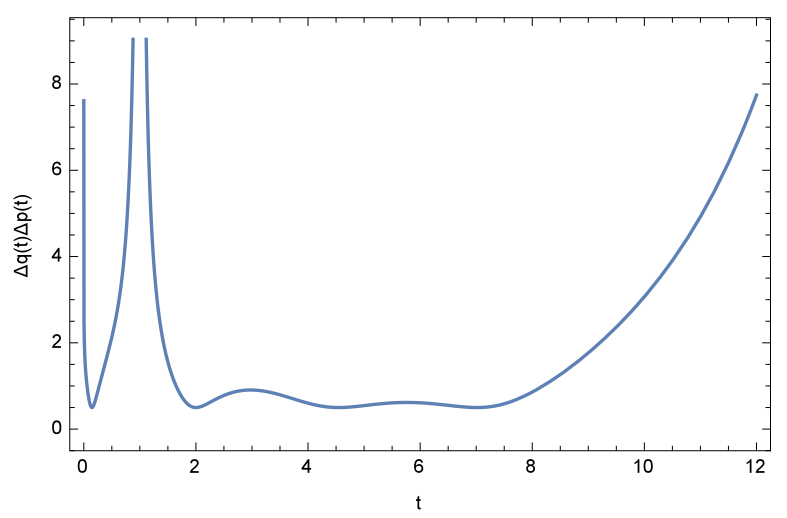

FIG. 5. Uncertainty relation for generalized Laguerre oscillator, $n=r=1$.

For $t_{0}=2$, solutions $x_{1}(t)$ and $x_{2}(t)$ of Eq. (71) are same as in part (a), and the particular solution satisfying the initial conditions $x_{p}(2)=0, \dot{x}_{p}(2)=e^{2}$, is $x_{p}(t)=(2-t) L_{1}(t) e^{t}$. Thus, the new probability density is

$$
\begin{aligned}
\rho_{k}^{1,1}(q, t)= & N_{k}^{2} R_{B}(t) \exp \left(-\frac{m \omega_{0}}{\hbar} R_{B}^{2}(t)\left(q+(t-2) L_{1}(t) e^{t}\right)^{2}\right) \\
& \times H_{k}^{2}\left(\sqrt{\frac{m \omega_{0}}{\hbar}} R_{B}(t)\left(q+(t-2) L_{1}(t) e^{t}\right)\right)
\end{aligned}
$$

with $R_{B}(t)$ as found in part (a), and position coordinate displaced by $x_{p}(t)$, see Fig. 6(a).

The new probability density follows the classical trajectory

$$
\langle\hat{q}\rangle_{\alpha}(t)=\sqrt{\frac{2 \hbar}{m \omega_{0}}}\left(\alpha_{1} x_{1}(t)+\alpha_{2}\left(m \omega_{0}\right) x_{2}(t)\right)+(2-t) L_{1}(t) e^{t},
$$

and it is plotted in Fig. 6(b). Comparing the probability densities, found in part (a) and part (b) of this example, one can explicitly see the change in the evolution of the wave packets under the displacement of the position coordinate by $x_{p}(t)$.

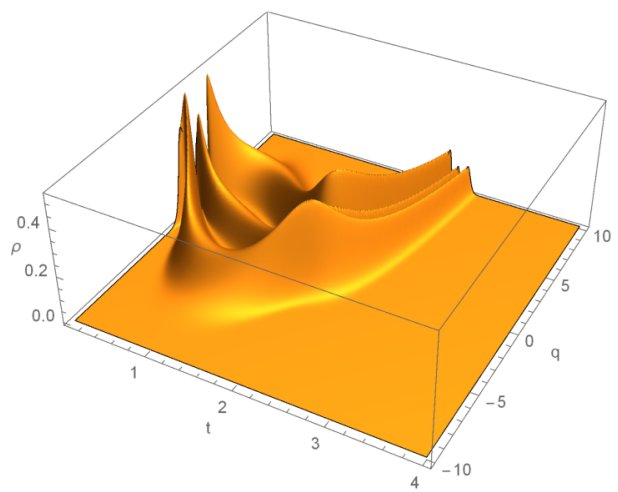

(a)

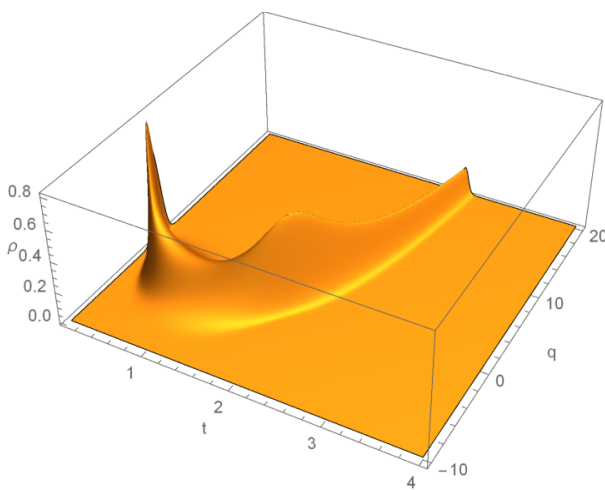

(b)

FIG. 6. Laguerre type generalized oscillator, when $D(t)=(t-2) L_{2}(t), E(t)=(1-t) L_{2}(t) e^{t}$. (a) Probability density $\rho_{2}^{1,1}(q, t)=\left|\Psi_{2}^{1,1}(q, t)\right|^{2}, n=r=1$, and $k=2$. (b) Probability density $\rho_{\alpha}^{1,1}(q, t)=\left|\Psi_{\alpha}^{1,1}(q, t)\right|^{2}$ in coherent states for $\alpha=$ $1 / \sqrt{2}+i(1 / \sqrt{2}), n=r=1$. 


\section{Jacobi type generalized quantum oscillator}

We define a generalized Jacobi type oscillator by a Hamiltonian of the form

$$
\begin{aligned}
\hat{H}_{g}(t) & =\frac{\hat{p}^{2}}{2(1-t)^{a+1}(1+t)^{a+1}}+\frac{[n(n+a+b+1)](1-t)^{a}(1+t)^{b}}{2} \hat{q}^{2}+\left(\frac{\dot{P}_{r}^{a, b}(t)}{P_{r}^{a, b}(t)}\right) \frac{(\hat{q} \hat{p}+\hat{p} \hat{q})}{2} \\
& +D(t) \hat{q}+E(t) \hat{p}+F(t),
\end{aligned}
$$

with mass $\mu(t)=(1-t)^{a+1}(1+t)^{b+1}, a, b>-1$, frequency $\omega^{2}(t)=[n(n+a+b+1)] /\left(1-t^{2}\right)$, $-1<t<1$, and $B(t)=\dot{P}_{r}^{a, b}(t) / P_{r}^{a, b}(t)$, where

$$
P_{n}^{a, b}(t)=\frac{(-1)^{n}}{2^{n} n !}(1-t)^{-a}(1+t)^{-b} \frac{d^{n}}{d t^{n}}\left[(1-t)^{a+n}(1+t)^{b+n}\right],
$$

are the Jacobi polynomials. Then, the corresponding classical oscillator is a forced Jacobi differential equation

$$
\begin{aligned}
& \ddot{x}+\frac{(b-a-(a+b+2) t)}{1-t^{2}} \dot{x}+\frac{n(n+a+b+1)+r(r+a+b+1)}{1-t^{2}} x \\
= & -\frac{1}{\mu} D+\dot{E}+\left(\frac{(b-a-(a+b+2) t)}{\left(1-t^{2}\right)}+\frac{\dot{P}_{r}^{a, b}(t)}{P_{r}^{a, b}(t)}\right) E, \quad-1<t<1,
\end{aligned}
$$

where $\Gamma(t)=[(b-a-(a+b+2) t)] /\left(1-t^{2}\right)$ is the damping coefficient, and

$$
\Omega^{2}(t)=\frac{n(n+a+b+1)}{1-t^{2}}+\frac{r(r+a+b+1)}{1-t^{2}}
$$

is the modified frequency. Thus, to preserve the structure after the modification, for given $n, r=$ $0,1,2, \ldots$ and $a, b>-1$, we need to find nonnegative integer $m$, for which the equation $n(n+$ $a+b+1)+r(r+a+b+1)=m(m+a+b+1)$ holds. We shall treat explicitly two special cases: for $a=b=0$ the Legendre generalized oscillators and for $a=b=-1 / 2$ the first-kind Chebyshev (FKC) oscillator.

\section{Legendre type generalized quantum oscillator}

The Hamiltonian for a Legendre type generalized oscillator is

$$
\hat{H}_{g}(t)=\frac{1}{2\left(1-t^{2}\right)} \hat{p}^{2}+\frac{n(n+1)}{2} \hat{q}^{2}+\left(\frac{\dot{P}_{r}(t)}{P_{r}(t)}\right) \frac{(\hat{q} \hat{p}+\hat{p} \hat{q})}{2}+D(t) \hat{q}+E(t) \hat{p}+F(t),
$$

where $\mu(t)=\left(1-t^{2}\right), \omega^{2}(t)=n(n+1) /\left(1-t^{2}\right), n=0,1,2, \ldots, t \in(-1,1), B(t)=\dot{P}_{r}(t) / P_{r}(t)$, and

$$
P_{r}(t)=\frac{1}{2^{r}} \sum_{k=0}^{\lfloor r / 2\rfloor}(-1)^{k} \frac{(2 r-2 k) !}{k !(r-k) !(r-2 k) !} t^{r-2 k}, r=0,1,2, \ldots
$$

are the Legendre polynomials. Then, the classical equation is a forced Legendre differential equation

$$
\ddot{x}-\frac{2 t}{1-t^{2}} \dot{x}+\frac{n(n+1)+r(r+1)}{1-t^{2}} x=-\frac{1}{\mu} D+\dot{E}+\left(-\frac{2 t}{1-t^{2}}+\frac{\dot{P}_{r}(t)}{P_{r}(t)}\right) E, \quad-1<t<1,
$$

with $\Gamma(t)=-2 t /\left(1-t^{2}\right)$ and $\Omega^{2}(t)=[n(n+1)+r(r+1)] /\left(1-t^{2}\right)$. Here, if for given $n$ and $r(r \neq$ $1), m$ is a positive integer satisfying the equation $n(n+1)+r(r+1)=m(m+1)$, then the homogeneous part of Eq. (75) has a solution of the form

$$
x(t)=c_{1} P_{m}(t)+c_{2} Q_{m}(t), \quad t \in(-1,1),
$$

where $P_{m}(t)$ are Legendre polynomials, and $Q_{m}(t)$ are the Legendre functions of the second kind given by the formula

$$
Q_{m}(t)=\frac{1}{2} P_{m}(t) \ln \frac{1+t}{1-t}-\sum_{k=1}^{m} \frac{1}{k} P_{k-1}(t) P_{m-k}(t) .
$$


Example 3. Let $n=2, r=2$, and $B(t)=\dot{P}_{2}(t) / P_{2}(t), D(t)=t P_{2}(t), E(t)=-P_{2}(t) / 6, F(t)=0$. Then the Hamiltonian becomes

$$
\hat{H}_{g}(t)=\frac{1}{2\left(1-t^{2}\right)} \hat{p}^{2}+3 \hat{q}^{2}+\left(\frac{\dot{P}_{2}(t)}{P_{2}(t)}\right) \frac{(\hat{q} \hat{p}+\hat{p} \hat{q})}{2}+t P_{2}(t) \hat{q}-\frac{P_{2}(t)}{6} \hat{p}
$$

and the corresponding classical equation is

$$
\ddot{x}+\frac{-2 t}{1-t^{2}} \dot{x}+\frac{12}{1-t^{2}} x=\frac{-1}{3}\left(\frac{2 t}{1-t^{2}} P_{2}(t)+\dot{P}_{2}(t)\right) .
$$

For $t_{0}=0$, two homogeneous solutions $x_{1}(t)$ and $x_{2}(t)$ of Eq. (76), satisfying the initial conditions $x_{1}(0)=-2 / 3, \dot{x}_{1}(0)=0$ and $x_{2}(0)=0, \dot{x}_{2}(0)=-3 / 2$, are

$$
x_{1}(t)=-Q_{3}(t)=\frac{5 t^{3}-3 t}{4} \ln \left(\frac{1-t}{1+t}\right)+\frac{5 t^{2}}{2}-\frac{2}{3}, \quad x_{2}(t)=P_{3}(t)=\frac{1}{2}\left(5 t^{3}-3 t\right),
$$

since for $n=2, r=2$ we have $m=3$. Then the particular solution satisfying the initial conditions $x_{p}(0)=0, \dot{x}_{p}(0)=1 / 12$, is

$$
x_{p}(t)=-\frac{t}{6} P_{2}(t)=-\frac{t}{12}\left(3 t^{2}-1\right)
$$

and we calculate

$$
R_{B}(t)=\frac{2}{3} \sqrt{\frac{1}{Q_{3}^{2}(t)+\left(\frac{4 m \omega_{0}}{9} P_{3}^{2}(t)\right)^{2}}},
$$

which is bounded and has oscillatory behavior for $t \in(-1,1)$, and $R_{B}(t) \rightarrow 0$, when $t \rightarrow \pm 1$.

Then, the probability density in state $\Psi_{k}^{2,2}(q, t)$ is

$$
\rho_{k}^{2,2}(q, t)=N_{k}^{2} R_{B}(t) \exp \left(-\frac{m \omega_{0}}{\hbar} R_{B}^{2}(t)\left(q+\frac{t P_{2}(t)}{6}\right)^{2}\right) H_{k}^{2}\left(\sqrt{\frac{m \omega_{0}}{\hbar}} R_{B}(t)\left(q+\frac{t P_{2}(t)}{6}\right)\right),
$$

which is plotted for $k=2$ in Fig. 7(a). Here

$$
\begin{aligned}
\langle\hat{q}\rangle_{\alpha}(t)= & \sqrt{\frac{2 \hbar}{m \omega_{0}}}\left[\alpha_{1} \frac{3 Q_{3}(t)}{2}-\alpha_{2}\left(\frac{2 m \omega_{0}}{3}\right) P_{3}(t)\right]-\frac{t P_{2}(t)}{6}, \\
\langle\hat{p}\rangle_{\alpha}(t)= & \sqrt{\frac{2 \hbar}{m \omega_{0}}}\left(1-t^{2}\right)\left[\frac{3}{2} \alpha_{1}\left(\dot{Q}_{3}(t)-\frac{\dot{P}_{2}(t)}{P_{2}(t)} Q_{3}(t)\right)\right. \\
& \left.+\alpha_{2}\left(\frac{2 m \omega_{0}}{3}\right)\left(\dot{P}_{3}(t)-\frac{\dot{P}_{2}(t)}{P_{2}(t)} P_{3}(t)\right)\right]
\end{aligned}
$$

and we plot the probability density $\rho_{\alpha}^{2,2}(q, t)$ in Fig. 7(b). With $R_{B}(t)$ given by (77) we get the fluctuation for $\hat{q}$ and $\hat{p}$, and uncertainty relation at coherent states as follows:

$$
\begin{aligned}
(\Delta \hat{q})_{\alpha}(t) & =\sqrt{\frac{\hbar}{2 m \omega_{0}}} \frac{1}{R_{B}(t)}, \\
(\Delta \hat{p})_{\alpha}(t) & =\sqrt{\frac{m \omega_{0} \hbar}{2}} R_{B}(t) \sqrt{1+\frac{\left(1-t^{2}\right)^{2}}{\left(m \omega_{0} R_{B}^{2}(t)\right)^{2}}\left(\frac{\dot{R}_{B}(t)}{R_{B}(t)}+\frac{\dot{P}_{2}(t)}{P_{2}(t)}\right)^{2}}, \\
(\Delta \hat{q})_{\alpha}(\Delta \hat{p})_{\alpha} & =\frac{\hbar}{2} \sqrt{1+\frac{\left(1-t^{2}\right)^{2}}{\left(m \omega_{0} R_{B}^{2}(t)\right)^{2}}\left(\frac{\dot{R}_{B}(t)}{R_{B}(t)}+\frac{\dot{P}_{2}(t)}{P_{2}(t)}\right)^{2}} .
\end{aligned}
$$

Because the coefficients of the forced oscillator (76) are continuous, the expectations (78) and fluctuations (80) of the position are smooth on the interval $t \in(-1,1)$. But the expectations (79) and fluctuations (81) of the momentum are not defined at zeros of $P_{2}(t)$. The uncertainty relation is bounded on $(-1,1)$, except in the neighborhoods of the zeros of $P_{2}(t)$, where it tends to infinity, see 


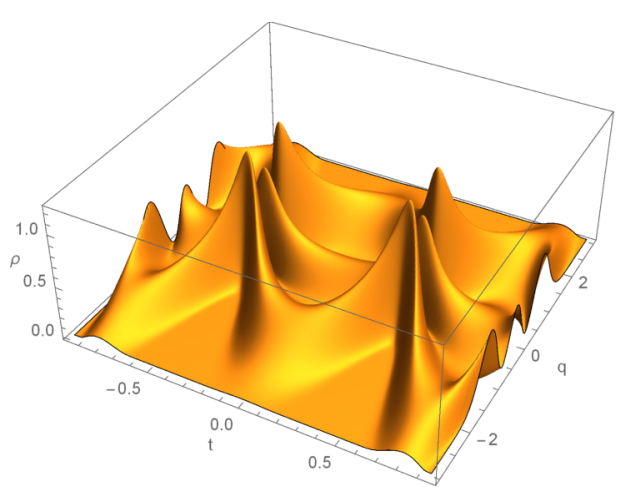

(a)

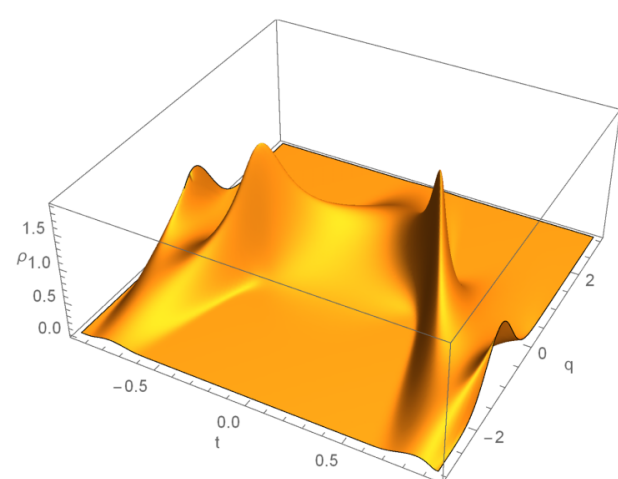

(b)

FIG. 7. Legendre type generalized oscillator, when $D(t)=t P_{2}(t), E(t)=-P_{2}(t) / 6, F(t)=0$. (a) Probability density $\rho_{2}^{2,2}(q, t)=\left|\Psi_{2}^{2,2}(q, t)\right|^{2}$. (b) Probability density $\rho_{\alpha}^{2,2}(q, t)=\left|\Phi_{\alpha}^{2,2}(q, t)\right|^{2}$ in coherent states for $\alpha=1 / \sqrt{2}+i(1 / \sqrt{2})$.

Fig. 8. Clearly, if the parameter $B(t)$ was taken to be zero, then the uncertainty relation would have been bounded for all $t \in(-1,1)$.

\section{First-kind Chebyshev type generalized quantum oscillator}

The Hamiltonian for a FKC generalized oscillator is

$$
\hat{H}_{g}(t)=\frac{\hat{p}^{2}}{2 \sqrt{1-t^{2}}}+\frac{n^{2}}{2 \sqrt{1-t^{2}}} \hat{q}^{2}+\left(\frac{\dot{T}_{r}(t)}{T_{r}(t)}\right) \frac{(\hat{q} \hat{p}+\hat{p} \hat{q})}{2}+D(t) \hat{q}+E(t) \hat{p}+F(t),
$$

where $\mu(t)=\sqrt{1-t^{2}}, \omega^{2}(t)=n^{2} /\left(1-t^{2}\right), n=0,1,2, \ldots, t \in(-1,1), B(t)=\dot{T}_{r}(t) / T_{r}(t)$, and

$$
T_{r}(t)=\frac{r}{2} \sum_{k=0}^{\lfloor r / 2\rfloor}(-1)^{k} \frac{(r-k-1) !}{k !(r-2 k) !}(2 t)^{r-2 k}, r=0,1,2, \ldots
$$

are the first-kind Chebyshev polynomials. Then, the classical equation is a forced FKC differential equation

$$
\ddot{x}-\frac{t}{1-t^{2}} \dot{x}+\frac{\left(n^{2}+r^{2}\right)}{1-t^{2}} x=-\frac{1}{\mu} D+\dot{E}+\left(-\frac{t}{1-t^{2}}+\frac{\dot{T}_{r}(t)}{T_{r}(t)}\right) E, \quad-1<t<1,
$$

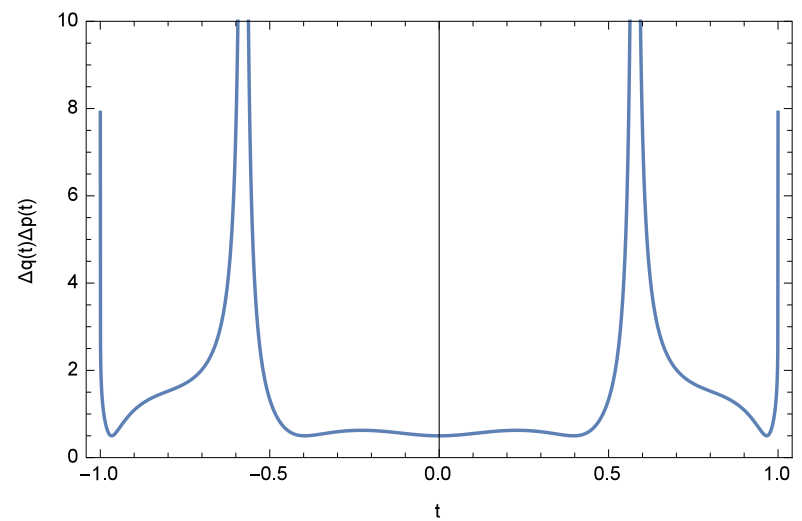

FIG. 8. Uncertainty relation for generalized Legendre oscillator, $n=r=2$. 


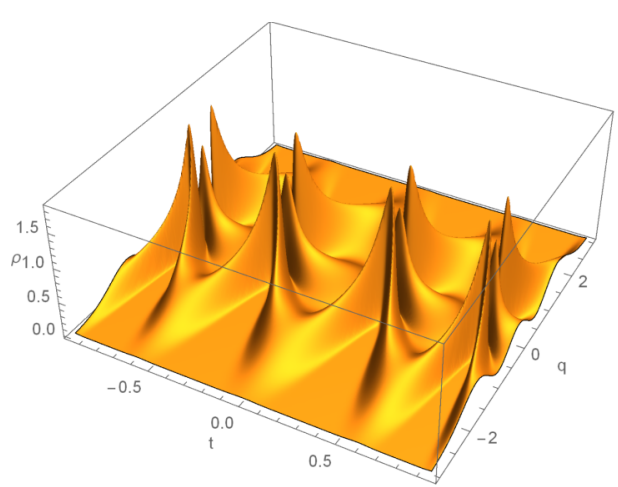

(a)

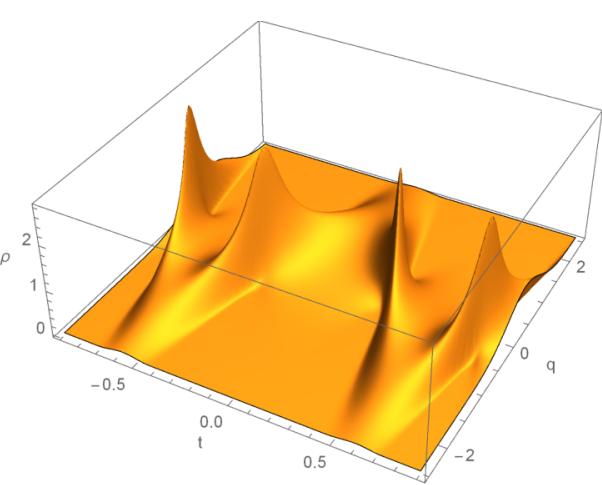

(b)

FIG. 9. FKC type generalized oscillator, when $D(t)=t T_{4}(t), E(t)=T_{6}(t)\left(2 t^{2}-1\right) /\left(64 \sqrt{1-t^{2}}\right), F(t)=0$. (a) Probability density $\rho_{2}^{3,4}(q, t)=\left|\Psi_{2}^{3,4}(q, t)\right|^{2}$. (b) Probability density $\rho_{\alpha}^{3,4}(q, t)=\left|\Phi_{\alpha}^{3,4}(q, t)\right|^{2}$ in coherent states for $\alpha=1 / \sqrt{2}+i(1 / \sqrt{2})$.

with $\Gamma(t)=-t /\left(1-t^{2}\right)$ and $\Omega^{2}(t)=\left(n^{2}+r^{2}\right) /\left(1-t^{2}\right)$. We note that, when $n^{2}+r^{2}=m^{2}$, where $m$ is also a positive integer, that is when $(n, r, m)$ are Pythagorean triples, the corresponding homogeneous equation has a solution of the form

$$
x(t)=c_{1} T_{m}(t)+c_{2} \sqrt{1-t^{2}} U_{m-1}(t),
$$

where

$$
U_{m}(t)=\sum_{k=0}^{\lfloor m / 2\rfloor}(-1)^{k} \frac{(m-k) !}{k !(m-2 k) !}(2 t)^{m-2 k}, m=0,1,2, \ldots
$$

are the Chebyshev polynomials of the second kind.

Example 4. Let $n=3, r=4, B(t)=\dot{T}_{4}(t) / T_{4}(t), D(t)=t T_{4}(t), E(t)=T_{6}(t)\left(2 t^{2}-1\right) /\left(64 \sqrt{1-t^{2}}\right)$, $F(t)=0$. Then, the Hamiltonian becomes

$$
\hat{H}_{g}(t)=\frac{\hat{p}^{2}}{2 \sqrt{1-t^{2}}}+\frac{9}{2 \sqrt{1-t^{2}}} \hat{q}^{2}+\left(\frac{\dot{T}_{4}(t)}{T_{4}(t)}\right) \frac{(\hat{q} \hat{p}+\hat{p} \hat{q})}{2}+t T_{4}(t) \hat{q}+\frac{T_{4}(t)}{9} \frac{\left(2 t^{2}-1\right)}{\sqrt{1-t^{2}}} \hat{p},
$$

and the corresponding classical equation is

$$
\ddot{x}-\frac{t}{1-t^{2}} \dot{x}+\frac{5}{1-t^{2}} x=\frac{1}{9 \sqrt{1-t^{2}}}\left(5 t T_{4}(t)+2\left(2 t^{2}-1\right) \dot{T}_{4}(t)\right),
$$

where by the above choice of $E(t)$, the singularities in $B(t)$ are removed, so that the forcing in Eq. (85) becomes continuous. For $t_{0}=0$, homogeneous solutions $x_{1}(t)$ and $x_{2}(t)$ of the Eq. (85), satisfying the initial conditions $x_{1}(0)=1 / 5, \dot{x}_{1}(0)=0$, and $x_{2}(0)=0, \dot{x}_{2}(0)=5$, respectively, are

$$
x_{1}(t)=\frac{1}{5} \sqrt{1-t^{2}} U_{4}(t)=\frac{1}{5} \sqrt{1-t^{2}}\left(16 t^{4}-12 t^{2}+1\right), \quad x_{2}(t)=T_{5}(t)=16 t^{5}-20 t^{3}+5 t,
$$

and the particular solution satisfying the initial conditions $x_{p}(0)=0, \dot{x}_{p}(0)=-1 / 9$ is

$$
x_{p}(t)=-\frac{t}{9} \sqrt{1-t^{2}} T_{4}(t)=-\frac{t}{9} \sqrt{1-t^{2}}\left(8 t^{4}-8 t^{2}+1\right) .
$$

Then, we calculate

$$
R_{B}(t)=\sqrt{\frac{1}{\left(1-t^{2}\right) U_{4}^{2}(t)+\frac{1}{25}\left(m \omega_{0} T_{5}(t)\right)^{2}}},
$$

where $R_{B}(t)$ is bounded and oscillating in $t \in(-1,1)$, but does not approach zero for $t \rightarrow \pm 1$, as in the case of the Legendre oscillator, since Chebyshev polynomials are defined at $t= \pm 1$. Then, the 


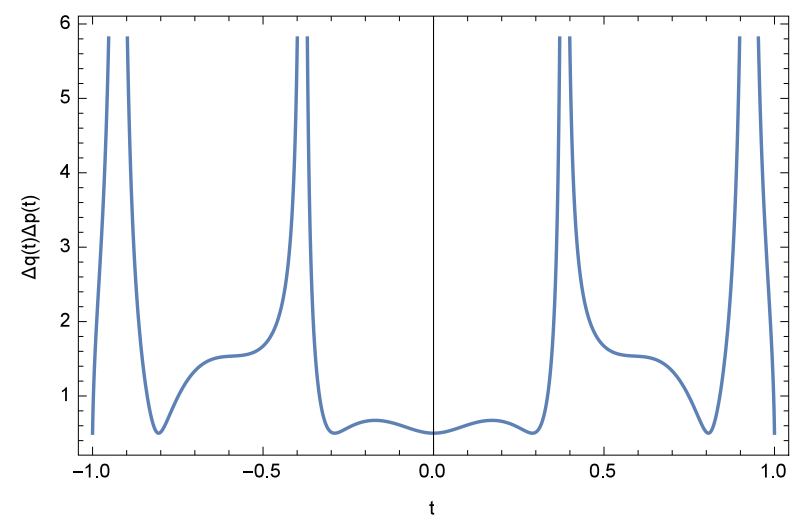

FIG. 10. Uncertainty relation for generalized FKC oscillator, $n=3, r=4$.

probability density for $n=3, r=4$ is in the form

$$
\begin{aligned}
\rho_{k}^{3,4}(q, t)= & N_{k}^{2} R_{B}(t) \exp \left(-\left(\sqrt{\frac{m \omega_{0}}{\hbar}} R_{B}(t)\left(q+\frac{t \sqrt{1-t^{2}}}{9} T_{4}(t)\right)\right)^{2}\right) \\
& \times H_{k}^{2}\left(\sqrt{\frac{m \omega_{0}}{\hbar}} R_{B}(t)\left(q+\frac{t \sqrt{1-t^{2}}}{9} T_{4}(t)\right)\right),
\end{aligned}
$$

and in Fig. 9(a) we plot it for $k=2$. In this example, we found the expectation values as

$$
\begin{aligned}
\langle\hat{q}\rangle_{\alpha}(t)= & \sqrt{\frac{2 \hbar}{m \omega_{0}}}\left[\alpha_{1} \sqrt{1-t^{2}} U_{4}(t)+\alpha_{2}\left(\frac{m \omega_{0}}{5}\right) T_{5}(t)\right]-\frac{t \sqrt{1-t^{2}}}{9} T_{4}(t), \\
\langle\hat{p}\rangle_{\alpha}(t)= & \sqrt{\frac{2 \hbar}{m \omega_{0}}}\left\{\frac{\alpha_{1}}{5}\left[\left(1-t^{2}\right) \dot{U}_{4}(t)-\left(t+\left(1-t^{2}\right) \frac{\dot{T}_{4}(t)}{T_{4}(t)}\right) U_{4}(t)\right]\right. \\
& \left.+\alpha_{2}\left(\frac{m \omega_{0}}{5}\right) \sqrt{1-t^{2}}\left(\dot{T}_{5}(t)-\frac{\dot{T}_{4}(t)}{T_{4}(t)} T_{5}(t)\right)\right\},
\end{aligned}
$$

and we plot $\rho_{\alpha}^{3,4}(q, t)$ in Fig. 9(b). Also, with $R_{B}(t)$ given by (86) we have

$$
\begin{aligned}
(\Delta \hat{q})_{\alpha}(t) & =\sqrt{\frac{\hbar}{2 m \omega_{0}}} \frac{1}{R_{B}(t)}, \\
(\Delta \hat{p})_{\alpha}(t) & =\sqrt{\frac{m \omega_{0} \hbar}{2}} R_{B}(t) \sqrt{1+\frac{1-t^{2}}{\left(m \omega_{0} R_{B}^{2}(t)\right)^{2}}\left(\frac{\dot{R}_{B}(t)}{R_{B}(t)}+\frac{\dot{T}_{4}(t)}{T_{4}(t)}\right)^{2}}, \\
(\Delta \hat{q})_{\alpha}(\Delta \hat{p})_{\alpha} & =\frac{\hbar}{2} \sqrt{1+\frac{1-t^{2}}{\left(m \omega_{0} R_{B}^{2}(t)\right)^{2}}\left(\frac{\dot{R}_{B}(t)}{R_{B}(t)}+\frac{\dot{T}_{4}(t)}{T_{4}(t)}\right)^{2}} .
\end{aligned}
$$

We see that the expectations (87) and fluctuations (89) of the position are smooth, but at the singularities of $B(t)$ the expectations (88) and fluctuations (90) of momentum are not defined. Since $B(t)$ has singularities at the four zeros of the FKC polynomial $T_{4}(t)$, the uncertainty relation is also singular at these points. On the other hand, when $|t| \rightarrow \pm 1$, uncertainty approaches minimum, that is $(\Delta \hat{q})_{\alpha}(\Delta \hat{p})_{\alpha}(t) \rightarrow \hbar / 2$, see Fig. 10 .

\section{SUMMARY AND DISCUSSION}

Quantum system with the most general quadratic Hamiltonian and time-variable parameters was solved using Wei-Norman algebraic approach. We found the exact form of the evolution 
operator in terms of two linearly independent homogeneous solutions and a particular solution of the corresponding forced classical oscillator. Using the evolution operator we were able to obtain wave functions, coherent states, probability densities, expectations, and uncertainties. These general results can be directly used to study many limiting cases and various variable parametric oscillators.

To understand better the behavior of such systems, it is always important to have exactly solvable models. A large class of exactly solvable harmonic oscillator models related with the classical orthogonal polynomials and special functions was introduced in Ref. 33. As an extension and generalization of these results, in the present work we have investigated quantum parametric oscillators of Hermite, Laguerre, and Jacobi type, under the influence of external forces. By a special choice of the mixed term parameter $B(t)$, which modifies the original frequency, we were able to preserve the structure of the original oscillator. However, as we have seen in Hermite, Laguerre, and Jacobi type oscillators, this choice of $B(t)$ develops finite time singularities at the zeros of the related orthogonal polynomials. In that case, we observed that probability densities and expectations of position remain smooth, but singularities of $B(t)$ are reflected in the expectations of momentum and the uncertainty relation. Precisely, momentum is not defined at these points, and when time approaches these singularities, uncertainty relation tends to infinity.

The probability densities of all models in consideration are showing oscillatory behavior in some finite time interval near the initial point, due to finite number of oscillations in the corresponding classical oscillator. When time increases, we have studied the contribution of $B(t)$ to the amplitude and the spreading coefficient $R_{B}(t)$ of the wave packets, corresponding to wave functions $\Psi_{k}(q, t)$ and coherent states $\Phi_{\alpha}(q, t)$. For the Hermite and Laguerre oscillators, which are defined on infinite time intervals, the amplitude of the wave packets is decreasing and approaching zero with increasing time, and wave packets are spreading along $q$-coordinate, since $R_{B}(t) \rightarrow 0$ as $|t| \rightarrow \infty$. So, we can say that Hermite and Laguerre oscillators exhibit the opposite behavior to the Caldirola-Kanai oscillator with positive damping coefficient, for which probability density amplitudes increase without bound, and wave packets are squeezing as time increases, since $R_{B}(t) \rightarrow \infty$ as $t \rightarrow \infty .{ }^{45}$ For the Legendre oscillator, defined on finite time interval $(-1,1)$, we have $R_{B}(t) \rightarrow 0$, as $t \rightarrow \pm 1$, so that wave amplitudes approach zero in the neighborhood of $t= \pm 1$, and wave packets are spreading with respect to q. However, for the first-kind Chebyshev model, $R_{B}(t)$ is bounded in $t \in(-1,1)$, but does not approach zero for $t \rightarrow \pm 1$, as in the case of the Legendre oscillator.

On the other hand, we have confirmed that the parameters $D(t)$ and $E(t)$ of the linear terms lead to displacement in the position coordinate of the wave packets, so that expectation values of position and momentum are shifted by the particular solutions $x_{p}(t)$ and $p_{p}(t)$, of the classical oscillators. In order to see the influence of the external terms and make comparison, we have constructed several examples with and without external forces. The probability densities for each case were obtained, and their evolution was illustrated graphically. As we have seen in Hermite and Laguerre oscillators, the influence of the external linear terms can change the evolution of the wave packets essentially. The forces in the given models were chosen to be continuous, but it could be interesting to consider discontinuous and Dirac-delta like forces as well. The work in this direction is in progress.

Clearly, the problems elaborated in this work can provide a basis for investigation of various generalized parametric oscillators related with other orthogonal polynomials and hypergeometric functions. Moreover, the representation of the complex wave functions in terms of modulus and phase, $\Psi=\sqrt{\rho} \exp (i S / \hbar)$, transforms the linear Schrödinger equation into a system of nonlinear hydrodynamic-like equations, known as the Madelung fluid equations. ${ }^{45-47}$ Then, the Madelung representation of the generalized parametric oscillators can be used also as a fundamental tool for description of quantum fluids and nonlinear evolution equations with variable parameters. Such problems are now under investigation.

\section{ACKNOWLEDGMENTS}

The authors would like to thank Professor Oktay K. Pashaev for valuable discussions and comments.

${ }^{1}$ R. K. Colegrave and M. S. Abdalla, Opt. Acta: Int. J. Opt. 28, 495 (1981).

${ }^{2}$ I. A. Pedrosa, A. Rosas, and I. Guedes, J. Phys. A: Math. Gen. 38, 7757 (2005). 
${ }^{3}$ G. Dattoli, A. Torre, and J. C. Gallardo, Riv. Nuovo Cimento Ser. 11, 1 (1998).

${ }^{4}$ A. B. Nassar, Phys. Lett. A 106, 43 (1984).

${ }^{5}$ A. Malkin, V. I. Manko, and D. A. Trifonov, Phys. Lett. A 30(7), 414 (1969).

${ }^{6}$ A. Sakharov, Zh. Eksp. Teor. Fiz. 49, 345 (1965) [Sov. Phys. JETP 22, 241 (1966)].

${ }^{7}$ W. P. Schleich and H. Walther, Elements of Quantum Information (Wiley-Verlag, Weinheim, 2007).

${ }^{8}$ M. S. Sarandy, E. I. Duzzioni, and R. M. Serra, Phys. Lett. A 375, 3343 (2011).

${ }^{9}$ P. Caldirola, Nouovo Cimento 18, 393 (1941).

${ }^{10}$ E. Kanai, Prog. Theor. Phys. 3, 440 (1948).

${ }^{11}$ H. Dekker, Classical and Quantum Mechanics of Damped Harmonic Oscillator (North-Holland Publishing Company, Amsterdam, 1981), Physics Reports, Vol. 80, pp. 1-112.

12 K. Husimi, Prog. Theor. Phys. 9(4), 381 (1953).

13 R. Feynman, Phys. Rev. 84, 108 (1951).

${ }^{14}$ H. R. Lewis, Jr. and W. B. Riesenfeld, J. Math. Phys. 10, 1458 (1969).

15 J. Wei and E. Norman, J. Math. Phys. 4, 575 (1963).

16 A. I. Baz', Ya. B. Zel'dovic, and A. M. Perelomov, Scatering Reactions and Decays in Nonrelativistic Quantum Mechanics (Nauka, Moscow, 1971) (in Russian).

${ }^{17}$ I. A. Malkin and V. I. Manko, Dynamical Symmetries and Coherent States of Quantum Systems (Nauka, Moscow, 1979) (in Russian).

18 A. Perelomov, Generalized Goherent States and Their Applications (Springer-Verlag, 1986).

19 A. M. Perelomov and Ya. B. Zel'dovic, Quantum Mechanics. Selected Topics (World Scientific, 1998).

${ }^{20}$ J. G. Hartley and J. R. Ray, Phys. Rev. D 25, 382 (1982).

${ }^{21}$ I. A. Pedrosa, Phys. Rev. A 55, 3219 (1997).

22 C. M. Dantas, I. A. Pedrosa, and B. Baseia, Braz. J. Phys. 22, 33 (1992).

${ }^{23}$ V. V. Dodonov and V. I. Man'ko, Phys. Rev. A 20(2), 550 (1979).

${ }^{24}$ D. C. Khandekar and S. V. Lawande, J. Math. Phys. 20(9), 1870 (1979).

${ }^{25}$ K. H. Yeon, D. H. Kim, C. I. Um, T. F. George, and L. N. Pandey, Phys. Rev. A 55(6), 4023 (1997).

${ }^{26}$ K. H. Yeon, D. H. Kim, S. Zhang, C. I. Um, S. K. Hong, and I. H. Kim, J. Korean Phys. Soc. 30(2), 506 (1997).

27 J. R. Choi, Pramana 62(1), 13 (2004).

${ }^{28}$ J. R. Choi and D.-W. Kim, J. Korean Phys. Soc. 45(6), 1426 (2004).

29 J. R. Choi and I. H. Nahm, Int. J. Theor. Phys. 46(1), 1 (2007).

${ }^{30}$ R. Cordero-Soto, E. Suazo, and S. K. Suslov, Ann. Phys. 325, 1884 (2010).

${ }^{31}$ K. H. Yeon, C. I. Um, and T. F. George, Phys. Rev. A 68, 052108 (2003).

${ }^{32}$ D.-Y. Song, Phys. Rev. A 59(4), 2616 (1999).

${ }^{33}$ Ş. A. Büyükaşık, O. K. Pashaev, and E. Ulaş-Tigrak, J. Math. Phys. 50, 072102 (2009).

${ }^{34}$ G. Dattoli, S. Solimeno, and A. Torre, Phys. Rev. A 34, 2646 (1986).

${ }^{35}$ G. Dattoli, F. Orsitto, and A. Torre, Phys. Rev. A 34(3), 2466 (1986).

${ }^{36}$ G. Dattoli, M. Richetta, and A. Torre, Phys. Rev. A 37(6), 2007 (1988).

${ }^{37}$ F. M. Fernandez, Phys. Rev. A 40, 41 (1989).

${ }^{38}$ C. M. Cheng and P. C. W. Fung, "The SU(2)Đ h(4) Hamiltonian," J. Phys. A: Math. Gen. 23, 75 (1990).

${ }^{39}$ C. F. Lo, Phys. Rev. A 43(1), 404 (1991).

${ }^{40}$ C. F. Lo, Il Nuovo Cimento D 13(10), 127 (1991).

${ }^{41}$ K. Fan, Y. Zheng, W. Ren, and S. Ding, Int. J. Quantum Chem. 107, 1355 (2007).

${ }^{42}$ E. Schrödinger, Naturwissenschaften 14, 664 (1926).

${ }^{43}$ R. J. Glauber, Phys. Rev. 131, 2766 (1963).

${ }^{44}$ M. M. Nieto and L. M. Simmons, Jr., Phys. Rev. D 20, 1321 (1979).

45 Ş. A. Büyükaşık and O. K. Pashaev, J. Math. Phys. 51, 122108 (2010).

${ }^{46}$ E. Madelung, Z. Phys. 40, 322 (1926).

${ }^{47}$ Ş. A. Büyükaşık and O. K. Pashaev, J. Math. Chem. 50, 2716 (2012). 